\title{
Radio Emission Physics in the Crab Pulsar
}

\author{
JEA N A. EILEK $\mathbf{K}^{1,2} \dagger$, AND TIMOTHY H. HA NKIN S $\mathbf{S}^{1,2}$ \\ ${ }^{1}$ Physics Department, New Mexico Tech, Socorro NM 87801 USA \\ ${ }^{2}$ National Radio Astronomy Observatory, Socorro NM 87801 USA
}

(Received ?; revised ?; accepted ?. - To be entered by editorial office)

We review our high-time-resolution radio observations of the Crab pulsar and compare our data to a variety of models for the emission physics. The Main Pulse and the LowFrequency Interpulse come from regions somewhere in the high-altitude emission zones (caustics) that also produce pulsed X-ray and $\gamma$-ray emission. Although no emission model can fully explain these two components, the most likely models suggest they arise from a combination of beam-driven instabilities, coherent charge bunching and strong electromagnetic turbulence. Because the radio power fluctuates on a wide range of timescales, we know the emission zones are patchy and dynamic. It is tempting to invoke unsteady pair creation in high-altitude gaps as source of the variability, but current pair cascade models cannot explain the densities required by any of the likely models. It is harder to account for the mysterious High-Frequency Interpulse. We understand neither its origin within the magnetosphere nor the striking emission bands in its dynamic spectrum. The most promising models are based on analogies with solar zebra bands, but they require unusual plasma structures which are not part of our standard picture of the magnetosphere. We argue that radio observations can reveal much about the upper magnetosphere, but work is required before the models can address all of the data.

PACS codes: To be chosen in the submission process

\section{Introduction}

High-energy emission in pulsars comes from high altitudes. Recent work shows that most optical, X-ray and $\gamma$-ray emission comes from caustic zones at mid-to-high altitudes in the star's magnetosphere or wind. The details, however, remain unclear. The highenergy exist over the star's magnetic poles. They may also coincide with current sheets either within or outside of the light cylinder. The highly nonlinear behavior of relativistic photon caustics, combined with uncertainties in the magnetic field structure close to the light cylinder, means that any of these possible emission zones can reproduce observed highenergy light curves. Because we do not know whether pulsed high-energy emission is due to synchrotron radiation, curvature radiation or inverse Compton scattering, we have few constraints on physical conditions within the high-energy emission zones. We need more information, and believe radio observations can help.

Radio emission in a large number of pulsars comes from lower altitudes, close to the magnetic axis. This geometry works for many pulsars, but fails for an interesting minority.

$\dagger$ Email address for correspondence: jeilek@aoc.nrao.edu

$\dagger$ We use "high-energy" to refer to optical, X-ray and $\gamma$-ray bands; as distinct from the radio band which is our focus in this paper. 
For those stars - including the pulsar in the Crab Nebula, the subject of this paper - the similarity of the radio and high-energy light curves strongly suggests the radio emission comes from the same high-altitude regions that create the high-energy emission. However, the geometrical models do not address the physical mechanisms by which the magnetospheric plasma produces the intense radio emission we observe. Many different emission mechanisms have been proposed, but none has been proven to be operating in the general pulsar population.

Our group has approached the question of pulsar radio emission mechanisms by carrying out detailed studies of one object, the Crab pulsar. This pulsar shines in radio at many phases throughout its rotation period, with seven components detectable in its mean profilet. At least three, and probably five, of these come from high altitude emission zones, spatially coincident with the high-energy emission zones in this star's magnetosphere. The high time resolution and broad spectral bandwidth of our observations show that different components have different temporal and spectral characteristics. We infer that more than one type of radio emission mechanism is taking place within the star's magnetosphere.

In this paper we collect and review our observational results, compare them to competing radio emission models, and from this comparison discuss what physical conditions exist in the high-altitude zones that emit both radio and high-energy pulsed emission. We begin in Sec. 2 by reviewing the basic picture of the rotating pulsar magnetosphere and its modern extension to the high-altitude transition to the wind zone. We focus on where in the extended magnetosphere the radio and high-energy emission originate. In Sec. 3 we lay out physical conditions that must exist (the "emission physics") if the magnetosphere is to produce intense radio emission. In Sec. 4 we set the stage for our discussion of the radio properties of the Crab. We present the different components of the mean radio

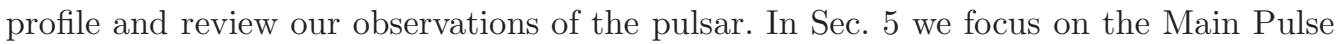
and the Low-Frequency Interpulse, which we believe involve similar emission physics. Both of these components show bursting behavior on sub-microsecond timescales; we argue this reflects variability of the driving mechanism, which is probably unshielded electric fields local to the region. We also introduce nanoshots - flares of emission on nanosecond timescales - which we believe reveal the fundamental emission process in these components. In Sec. 6 we compare observed nanoshot properties to predictions of three plausible radio emission models. Although none of the models can fully explain the data, we suggest they can be used to constrain physical conditions in the radio emission zones. We then switch to the High-Frequency Interpulse, a separate component with different radio characteristics that very likely involves different emission physics. In Sec. 7 we discuss its radio properties, including the unexpected spectral emission bands, and consider what emission physics might be responsible for the bands. In Sec. 8 we present two additional clues to its local environment: signal dispersion and polarization angle. Finally, in Sec. 9, after congratulating the steadfast reader who has made it to the end, we end by summarizing the lessons we have learned to help us going forward.

We relegate to the Appendices necessary details on a variety of possible radio emission mechanisms. In App. @ we briefly review specific models of coherent radio emission that have been proposed for the general pulsar population, and in App. B we review one

$\dagger$ We use the term "mean profile" to describe the radio intensity as a function of rotation phase. This quantity is derived by summing the received intensity synchronously with the star's rotation period. The same data product is often called the "light curve", especially in the high-energy community. 


$\begin{array}{ccccc}\text { altitude } & B & \nu_{\mathrm{B}} & n_{\mathrm{GJ}} & \nu_{\mathrm{p}}\left(n_{\mathrm{GJ}}\right) \\ R_{*} \simeq 10 \mathrm{~km} & 4 \times 10^{12} \mathrm{G} & 1 \times 10^{19} \mathrm{~Hz} & 8 \times 10^{12} \mathrm{~cm}^{-3} & 3 \times 10^{10} \mathrm{~Hz} \\ R_{\mathrm{LC}} / 2 \simeq 80 R_{*} & 8 \times 10^{9} \mathrm{G} & 2 \times 10^{16} \mathrm{~Hz} & 2 \times 10^{7} \mathrm{~cm}^{-3} & 4 \times 10^{7} \mathrm{~Hz} \\ R_{\mathrm{LC}} \simeq 160 R_{*} & 1 \times 10^{6} \mathrm{G} & 3 \times 10^{12} \mathrm{~Hz} & 2 \times 10^{6} \mathrm{~cm}^{-3} & 1 \times 10^{7} \mathrm{~Hz}\end{array}$

TABLE 1. Scaling numbers for the Crab pulsar, assuming the Goldreich-Julian ("GJ", Sec. 2) model holds. $\nu_{\mathrm{B}}$ is the lepton Larmor frequency; $\nu_{\mathrm{p}}$ is the lepton plasma frequency. Values are given at the star's surface and at two higher altitudes more relevant to the radio and high-energy emission. $R_{*} \simeq 10 \mathrm{~km}$ is the radius of the neutron star, which rotates at $\Omega_{*} \simeq 190 \mathrm{rad} / \mathrm{s}$ (rotation period $33 \mathrm{msec}$ ). The distance $R_{\mathrm{LC}}=c / \Omega_{*} \simeq 160 R_{*}$ is the light cylinder radius. The magnetic field is assumed to be dipolar. The number density of charge required to maintain corotation is $n_{\mathrm{GJ}} \simeq \Omega_{*} B / 2 \pi c e$. In many applications the number density of the plasma is thought to be enhanced relative to the GJ value, by a factor $\lambda=n / n_{\mathrm{GJ}}$.

additional model specifically proposed for the High-Frequency Interpulse of the Crab pulsar.

\section{Emission zones in the pulsar magnetosphere}

Consider a rapidly rotating neutron star which supports a strong magnetic field $\mathbb{\# \text { above }}$ the star's surface. Within the so-called "light cylinder" - the radius at which the corotation speed becomes lightspeed - the magnetic field geometry is thought to be nearly that of a vacuum dipole, rotating with the star and having its magnetic moment oriented at some oblique angle to the star's rotation axis. Approaching the light cylinder, relativistic effects "sweep back" the dipolar field lines, generating a toroidal component which becomes an outward propagating EM wave past the light cylinder (Deutsch 1955). Most poloidal field lines which leave the star's surface return to the pulsar well within the light cylinder, creating the "closed field zone". It is generally thought that plasma in this region, pulled from the star's surface by rotation-induced electric fields, becomes charge separated in just the amount needed to short out any parallel electric field. This necessary charge density is known as the "Goldreich-Julian charge density", $n_{\mathrm{GJ}}=\Omega_{*} \cdot \mathbf{B} / 2 \pi e c$, from a seminal ansatz by Goldreich \& Julian (1969, "GJ"). If this density is attained, it supports corotation of a force-free plasma magnetosphere in the closed-field-line region. It is also generally agreed that magnetic field lines or flux ropes which originate near the star's magnetic poles cross the light cylinder before they return to the star, creating an "open field line" region. The area on the star's surface traced by the outermost open field lines is called the "polar cap".

This model is hardly the final word on the subject. The structure of the magnetosphere is complex, dynamic and nonlinear. It is far from clear that it will ever attain the steadystate situation envisaged by Goldreich \& Julian, because magnetic fields, currents and charges feed back on each other, creating a very dynamic system. Observations reveal that the radio emission is variable on both short and long timescales; this also suggests that the emission regions are unsteady and dynamic. Nonetheless, the model provides valuable insights, and it has become part of the "standard pulsar picture" over the years. To illustrate the model, and for later reference for the Crab pulsar, we store important scaling numbers in Table 1.

$\dagger$ The surface magnetic field strength is inferred from the rate at which star's rotation slows down. The loss of rotational energy is ascribed to magnetic dipole radiation. Typical values are $B_{*} \sim 10^{12}-10^{13} \mathrm{G}$, with a dipolar $1 / r^{3}$ falloff to higher altitudes. 


\subsection{The traditional radio geometry: lighthouse beams}

The picture just described has been particularly successful in providing a context for understanding the pulsed radio emission by which most pulsars have been discovered. Plasma/current flow out along the open field lines enables the buildup of charge-starved regions (so-called "gaps"). Such gaps may fill the open field line region close to the star's surface (polar gap; e.g., Ruderman \& Sutherland 1975), or may sit along the edge of the open field line region (slot gap; Arons \& Scharlemann 1979). Parallel electric fields ( $E_{\|}$; component along B) in these gaps accelerate outflowing charges to very high Lorentz factors (typically $\gamma \sim 10^{6}-10^{7}$ ). Curvature radiation from these fast charges supports magnetic pair creation in the strong magnetic field close to the polar cap. The pair cascade enhances the plasma density, relative to $n_{\mathrm{GJ}}$, by a factor $\lambda=n / n_{\mathrm{GJ}}$. Models suggest $\lambda \sim 10^{2}-10^{4}$, and a final pair streaming speed $\gamma_{s} \sim 10^{2}-10^{3}$ (Hibschman \& Arons 2001, Arendt \& Eilek 2002). Thus, result of the pair cascade is a fast particle beam moving through a slower-moving pair plasma, both still moving out along the open field lines.

If some part of the energy in the fast outflow through the open field line region is converted to radio emission - which will be strongly forward beamed, approximately along the star's magnetic axis - an observer will see a radio pulse every time the star's rotation sweeps this "lighthouse beam" past his or her sightline. In this simple picture, most pulsars should show only one pulse per rotation period, which is indeed the case. We would need special geometry to see more: either the magnetic moment and the viewing angle are both at $90^{\circ}$ to the rotation axis (giving two pulses $180^{\circ}$ apart), or both are very close to the rotation axis (giving a complex pulse distribution if the emitting region is inhomogeneous). This model also predicts the position angle of linear polarization should rotate as the radio beam crosses the observer's sightline (Radhakrishnan \& Cooke 1969; assuming the polarization direction is rigidly tied to magnetic field geometry in the emission zone). Because both predictions hold true for the majority of well-studied pulsars, this geometry has become part of the standard pulsar picture for the past several decades.

\subsection{Expand this picture: the extended magnetosphere}

We've learned much more in recent years. The happy conjunction of abundant highenergy pulsar observations with significant advances in numerical simulations (both MHD and PIC methods) have greatly broadened our understanding of the high-altitude magnetosphere and its transition to the pulsar wind (known as the "extended" magnetosphere; e.g., Kalapotharakos et al. 2012a). Various analytic attempts to understand the region around the light cylinder generally proved less than successful over the years, but several groups have now modelled this region numerically. Initial numerical work assumed pair creation was sufficiently abundant that the force-free condition $(\mathbf{E} \cdot \mathbf{B}=0)$ holds everywhere (e.g., Contopoulos \& Kalapotharakos 2010, Bai \& Spitkovsky 2010). More recently, modelling has been extended to include phenomenological resistivity in regions where current sheets are expected (e.g., Kalapotharakos et al. 2012b, Li et al. 2012 ).

While details differ between authors, the basic properties of the extended magnetosphere seem agreed on, and are in good agreement with the analytic models of Bogovalov (1999). Closed field lines and charge-separated regions exist within light cylinder, as pre-

$\ddagger$ In magnetic fields close to the critical field, (the field at which quantum effects become important, $B_{c}=m^{2} c^{3} / e \hbar \sim 4.4 \times 10^{13} \mathrm{G}$ ) a single energetic photon can create an electron-positron pair. Radiation from the daughter leptons in turn creates more pairs, which create more photons, and so on ... leading to a pair creation cascade. 
dicted by the GJ model. Plasma flows out open field lines, crosses the light cylinder, developing into an outflowing pulsar wind. Past the light cylinder, the plasma flow which must remain sublight - trails the star's rotation, creating a spiral pattern for the toroidal magnetic field. The poloidal field becomes asymptotically radial and develops a "split monopole" structure, changing sign across an undulating equatorial current sheet. This picture of the extended magnetosphere reveals more regions which may accelerate particles to relativistic energies and have the potential to be emission zones for high energy emission, radio emission, or both.

Gaps are regions where force-free MHD breaks down and unshielded $E_{\|}$can exist; a variety have been suggested. In addition to the low-altitude polar gaps described above, some authors have suggested slot gaps, which extend the polar gap from the star's surface out to the light cylinder along the edges of the open field line region (Arons \& Scharlemann 1979, Muslimov \& Harding 2004; also called two-pole caustics, see next section.) Other authors have suggested outer gaps and annular gaps: vacuum regions allowing $E_{\|} \neq 0$, originating close to the so-called neutral charge surface (partway out along the open field region, where the charge density needed for corotation changes sign), and extending by some distance both towards the light cylinder and into the polar flux tube (e.g., Cheng et al. 1986, Romani \& Yadigaroglu 1995, Qiao et al. 2004).

Current sheets have also been suggested as possible sites of particle energization and high-energy radiation. In models of the extended magnetosphere, current sheets exist both within the light cylinder - where currents flow along the separatrices between open and closed field lines - and external to the light cylinder - where an undulating current sheet, frozen into the wind, separates the two hemispheres of the split-monopole wind. It is often argued that reconnection events across the current sheets energize the plasma, possibly to relativistic energies, making them additional sources of high-energy emission (e.g., Petri \& Kirk 2005, Bai \& Spitkovsky 2010, Cerutti et al. 2015). Although we have not seen much discussion of these current sheets as sites of radio emission, we think the topic warrants further study.

\subsection{Caustics, sky maps and high-energy emission}

Rapid recent advances in high-energy pulsar observations - particularly the many $\gamma$-ray light curves obtained by Fermi (Abdo et al. 2013) - reveal that double-peaked highenergy pulsar light curves are the rule rather than the exception. This shows that highenergy emission cannot come from low-altitudes, because there are far too many doublepeaked high-energy light curves to be consistent with the special geometry needed (both viewing and inclination angles close to $90^{\circ}$ ) if the emission were from low-altitude polar gaps. It is now generally accepted that the two bright peaks typical of high-energy light curves come from extended high-altitude regions - gaps or current sheets - associated with but not local to the star's two magnetic poles.

The detailed structure of these high-altitude emission regions is not understood, and cannot easily be determined from observations. Finite photon light travel times, and the non-dipolar magnetic field geometry at high altitudes, cause outgoing photons emitted parallel to B to accumulate in a few preferred directions ("caustics"). Simulations document a highly nonlinear mapping between the spatial structure of magnetospheric emission regions and the rotation phases and viewing angles at which emitted radiation is caught by the observer. In particular, double-peaked light curves do not require special geometry, but are seen from a wide range of viewing and orientation angles (e.g, Dyks et al. 2004, Bai \& Spitkovsky 2010).

How does all this connect to radio emission? In many pulsars, the radio and highenergy light curves are out of phase, with the main radio pulse leading the brighter of 
the two high-energy pulses by $0.2-0.3$ of a rotation period. This is generally ascribed to radio emission coming from low-altitude polar gaps, while high-energy emission comes from high altitudes. Because the emission mechanisms are also different, one might think the radio and high-energy emission zones have little to do with each other.

The situation is different, however, for an interesting subset of radio pulsars, including the Crab pulsar. In these stars the main radio and high-energy peaks occur at the same rotation phases. This suggests that both the radio and high-energy emission come from the same spatial regions - high-altitude gaps or current sheets - sitting somewhere in the upper reaches of the magnetosphere. Although the location and structure of the emission regions are not yet understood, comparison of radio and high-energy profiles gives some insight. The two high-energy pulses of the Crab pulsar are much broader than their radio counterparts, and emission bridges between the peaks are seen at high energies but not in radio (e.g., Abdo et al. 2010). There is a modest correlation between bright radio pulses and enhanced optical pulses (Shearer et al. 2012). Putting this all together, we infer that radio emission sites in the Crab pulsar are related to high-energy emission sites, but are more localized. Furthermore, the radio emission is highly unsteady; it varies on short and long timescales. We infer that the radio emission sites are dynamic, inhomogeneous regions.

\section{How do pulsars shine in radio?}

Although the standard picture described in Sec. 2 can explain the phenomenology of most radio pulsars, details of the physics are not well addressed. The key question unanswered despite several decades of hard work - is just how and where the radio emission is produced. The problem is made challenging by the high brightness temperature $\rightarrow$ of the radio emission (often quoted as $10^{26} \mathrm{~K}$, but can be as high as $10^{41} \mathrm{~K}$; Hankins \& Eilek 2007). Such high intensities are unlikely to come from any incoherent emission process. Instead, a so-called "coherent" process is needed, such as maser amplification or coordinated motion of a group of charges.

Three things comprise the "emission physics" needed to make the intense radio emission we see from pulsars.

- There must be a robust, long-lived site within the magnetosphere where conditions are right to make the radio emission seen at regular, well-defined phases of the star's rotation ("pulses" or "components of the mean profile"). Polar gaps in the lighthouse model are one example; localized regions within extended high-altitude gaps or caustics are another example.

- There must be a source of available energy which can be tapped for radio emission; that source must come from dynamics within the magnetosphere. One example is relativistic plasma outflow in the open field line region, driven by electric fields parallel to the magnetic field. Another example may be conversion of magnetic energy, for instance reconnection events in current sheets.

- There must be a mechanism by which the available energy is converted to coherent radio emission. While it is generally agreed that such a mechanism must operate at the microphysical (plasma) scale, the details of the emission mechanism are not yet understood.

A wide range of models have been proposed, over the years, to meet these conditions

$\dagger$ The brightness temperature - a quantity easily measured by radiometers - measures the intensity at frequency $\nu$ of a radio beam: $k T_{\mathrm{B}}=I_{\nu} c^{2} / 2 \nu^{2}$. Brightness temperatures higher than any physically reasonable temperature point to coherent emission. 
and explain pulsar radio emission. None of them has yet emerged as the definitive answer, in part because it has been very hard to compare the models to the data. Most of the models assume a beam of relativistic charges provides the available energy, but other variants have also been suggested. There are great differences between the models in how that energy is converted to coherent radio emission, as well as in the plasma conditions required for a particular mechanism to work. We discuss several of these models in Appendix $\AA$ and test some of them against our data for the Crab pulsar in Secs. 6 and 7 below.

\section{Radio emission from the Crab pulsar}

The well-studied pulsar in the Crab Nebula is an important case study, because the high radio power of its occasional "giant" pulses makes it an ideal target for dedicated radio studies, such as our group has carried out over the years. We have studied the star over a wide frequency range, capturing individual bright pulses with time resolution as low a fraction of a nanosecond. We have also continuously sampled the star's radio emission at many frequencies, from which we determine both the star's mean radio profile and the unsteady nature of its radio emission. In this paper we draw on this body of work to characterize the Crab pulsar's radio properties and consider what they reveal about conditions in the pulsar's radio emission zones. The reader interested in further details of our work can refer to Moffett \& Hankins $(1996,1999)$; Hankins et al. (2003); Hankins \& Eilek (2007); Crossley et al. (2010); Hankins et al. (2015) and Hankins et al. (2016).

\subsection{Mean profile of the Crab pulsar}

The mean radio profile of the Crab pulsar is complex, with seven distinct components identified so far. The strength of each component depends on frequency: radio observations above $\sim 5 \mathrm{GHz}$ find a very different profile from that seen at lower radio frequencies. We show the radio evolution of the mean profile, with components labelled, in Fig. 1 and summarize the components in Table 2 .

The Crab's mean profile at low radio frequencies - between $\sim 100 \mathrm{MHz}$ and $\sim 5 \mathrm{GHz}-$ is dominated by two peaks, the Main Pulse and the Low Frequency Interpulse, separated by $\sim 145^{\circ}$ of rotation phase. Both are phase coincident with the two strong peaks in highenergy light curves (e.g., Abdo et al. 2010; Zampieri et al. 2014). However, both radio components are significantly narrower in phase than their high-energy counterparts, and each radio pulse lags the corresponding high-energy pulse by $\sim 2-3^{\circ}$ of phase. Two additional weak components can be seen at low radio frequencies: the Precursor leads the Main Pulse by $\sim 20^{\circ}$, and the Low-Frequency Component leads the Main Pulse by $\sim 37^{\circ}$.

The Crab's mean profile is quite different at high radio frequencies. It is dominated by an interpulse and two new components which are weak or nonexistent below $\sim 5$ GHz. The Main Pulse nearly disappears above $\sim 8 \mathrm{GHz}$. The Interpulse undergoes a discontinuous phase shift of $\sim 7^{\circ}$, and also undergoes a dramatic change in its spectral and temporal properties of its single pulses. Because the differences between the lowfrequency and high-frequency Interpulses are so striking, we identify them as two separate components. Despite the $7^{\circ}$ phase shift, the High-Frequency Interpulse is also phase coincident with the second of the two broad peaks in the high-energy light curve, but now this radio component leads the high-energy component by $\sim 3^{\circ}$ of phase.

Two new mean-profile components appear at high radio frequencies: the High-Frequency Components. These first appear at a few $\mathrm{GHz}$, and become increasingly dominant going to higher frequencies. By $28 \mathrm{GHz}$, the highest frequency at which we have a mean 


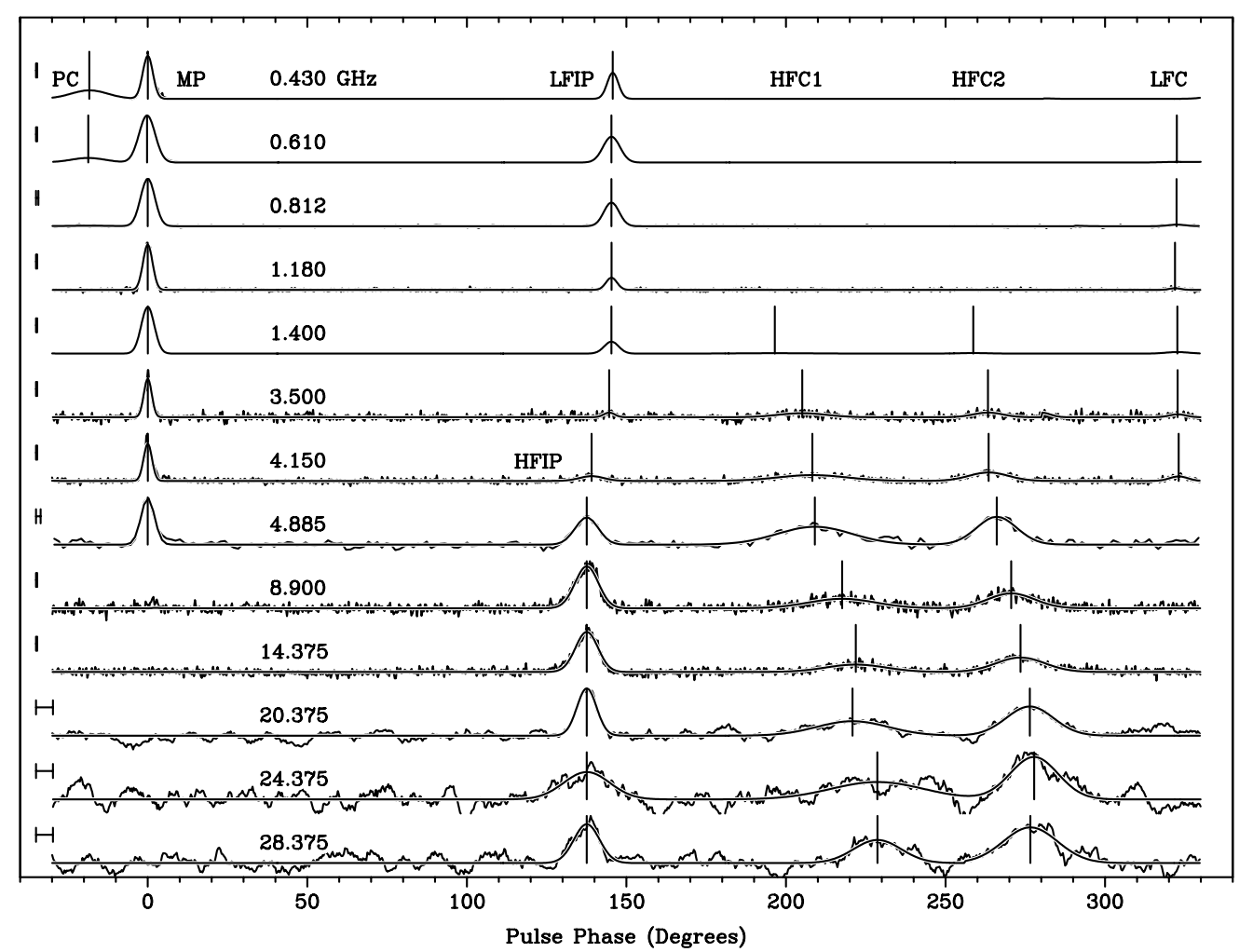

FiguRE 1. Mean radio profiles for the Crab pulsar, at a number of frequencies, with formal Gaussian fits overplotted. The dominant components discussed in this paper are described in the text and summarized with acronyms in Table 2. The Main Pulse (MP) and the two Interpulses (LFIP, HFIP) are coincident in phase with the two peaks in the high-energy profile, which suggests a similar spatial origin for the radio and high-energy emission. The High-Frequency Components have not been clearly detected in high energy profiles. Additional radio components seen at low frequencies, the Precursor (PC) and the Low-Frequency Component (LFC), may come from low-altitudes close to the star's polar cap. Nomenclature is from Moffett \& Hankins (1996); figure from Hankins et al. (2015).

profile, the two High-Frequency Components are as strong as the High-Frequency Interpulse. Unlike other components, the High-Frequency Components drift in rotation phase, appearing later in phase as one goes to higher frequencies. There is no clear sign of either High-Frequency Component in high-energy profiles, except for a weak possible detection in the $\gtrsim 10 \mathrm{GeV}$ profile of Abdo et al. (2010).

The fact that we see seven radio components, distributed throughout the full rotation period, immediately tells us we are not seeing low-altitude, polar cap emission. If that were the case, we would see at most two components, separated by $\sim 180^{\circ}$ of phase. Furthermore, both the magnetic inclination angle and viewing angle of the pulsar would have to be $\sim 90^{\circ}$ in order for two polar-cap components to be detected. That requirement disagrees with our known viewing angle of $\sim 60^{\circ}$ relative to the star's rotation axis (determined from the X-ray torus which surrounds the pulsar; Ng \& Romani 2004). We thus conclude - consistent with arguments in Sec. 2.3 - that the main radio pulses $\forall$ from the Crab pulsar come from high altitude regions in the magnetosphere.

$\dagger$ Possible exceptions are the Precursor and the Low-Frequency Component; one of both of these may come from polar-cap emission. See discussion in Hankins et al. (2016). 


$\begin{array}{cccc}\text { Component } & \text { Acronym } & \text { Frequency Range } & \text { High-energy counterpart? } \\ \text { Precursor } & \text { PC } & 0.3-0.6 \mathrm{GHz} & \text { none found } \\ \text { Low-Frequency Component } & \text { LFC } & 0.6-4.2 \mathrm{GHz} & \text { none found } \\ \text { Main Pulse } & \text { MP } & 0.1-4.9 \mathrm{GHz} & \text { yes, P1 } \\ \text { Low-frequency Interpulse } & \text { LFIP } & 0.1-3.5 \mathrm{GHz} & \text { yes, P2 } \\ \text { High-frequency Interpulse } & \text { HFIP } & 4.2-28 . \mathrm{GHz} & \text { yes, P2 } \\ \text { High-frequency Components } & \text { HFC1,2 } & 1.4-28 . \mathrm{GHz} & \text { weak or non-existent }\end{array}$

TABle 2. Components of the mean radio profile of the Crab pulsar which we discuss in this paper. The frequency range describes the range over which components have been found in mean profiles from our group (Moffett \& Hankins 1996, Hankins et al. 2015) or other work (e.g. Rankin et al. 1970). We have occasionally detected single Main Pulses and Low-Frequency Interpulses above the listed frequency ranges, but they are too rare to contribute to mean profiles. Acronyms are used in Fig. 1 The two peaks seen in high-energy profiles are P1 ("main pulse") and P2 ("interpulse"); they can be tracked continuously from optical (Słowikowska et al. 2009) to $\gamma$-rays (e.g. Abdo et al. 2010).

\subsection{Our single-pulse observations of the Crab pulsar}

Our main focus in this paper is what we have learned about individual Main Pulses and Interpulses from our high-time resolution observations of the Crab pulsar. This work was carried out at between 1993 and 1999 at the Karl G. Jansky Very Large Array, between 2002 and 2009 at the Arecibo Observatory, and from 2009 and 2011 at the Robert C. Byrd Green Bank Telescope. Details of our single-pulse observations and dataacquistion system are given in Hankins \& Eilek (2007) and Hankins et al. (2015). Our net "take" was 1440 bright single pulses above $\sim 1 \mathrm{GHz}$ with high enough signal-tonoise to be useful. Pulses at lower frequencies are significantly broadened by interstellar scintillation, thus not useful for our analysis here. We captured about 870 strong Main Pulses, 540 strong Interpulses (510 of which were High-Frequency Interpulses, the rest Low-Frequency Interpulses), and 30 High-Frequency Component pulses. In this paper we focus on the Main Pulse and the two Interpulses, for which our data allow useful comparisons to the models.

Because our data-acquisition system is triggered only by the brightest pulses, we have captured single pulses from the bright end of the pulse fluence distribution. These are sometimes called "giant pulses" in the literature. However, there is no evidence that these are any different from fainter, "normal" pulses that make up the majority of the star's pulsed radio emission. We therefore assume the pulses we have captured are good representatives of the emission physics for both high and low radio power.

Our single-pulse work shows that two very different types of radio emission physics exist in the magnetosphere of the Crab pulsar. As we show in the following sections, single Main Pulses and Low-Frequency Interpulses share the same temporal and spectral characteristics, while single High-Frequency Interpulses are very different. This result was totally unexpected. In Sec. 2 we argued that the Main Pulse and the two Interpulses should be associated with the star's two magnetic poles, probably from high-altitude caustics above each pole. However, because no pulsar model to date predicts any asymmetry between the north and south magnetic poles, there is no reason to expect the radio emission physics from the two regions to be different. And yet that seems to be the case.

We emphasize that we have not seen any secular changes in the properties of the Main Pulse or either Interpulse. The characteristics we summarize here are robust and constant, over the nearly 20 years we have observed this pulsar. In the rest of this paper 
we present the detailed characteristics of each type of radio component, and consider how well - if at all - existing models can explain the observations.

\section{Main Pulse emission: microbursts and nanoshots}

Although the Main Pulse and Low-Frequency Interpulse components of the mean profile are smooth and well-fit by Gaussians (as in Fig. 1), the story is very different when pulses are observed individually.

\subsection{Explosive bursts of radio emission}

The radio emission in a single Main Pulse or Low-Frequency Interpulse comes in $m i$ crobursts: abrupt releases of coherent radio emission with a characteristic time of a few microseconds. Fig. 2 shows two such examples. These bursts can occur anywhere in the probability envelope defined by the mean-profile component, which extends for several hundred microseconds (Fig. 11). The burst strength is highly variable. The strongest bursts are the most dramatic, but weak bursts are more common. Furthermore, detectable bursts occur in random groups that persist for a few minutes, between longer periods of radio silence.

The spectrum of a microburst is relatively broadband. As Fig. 2 shows, it fills our observing bandwidth, which was typically 2 or $4 \mathrm{GHz}$ for most of our observations, and extended to $8 \mathrm{GHz}$ for one data set. No lower frequency limit has been found for Main Pulse emission; that component can be seen in mean profiles well below $100 \mathrm{MHz}$ (e.g., Rankin et al. 1970). Main Pulses and Low-Frequency Interpulses become faint and/or scarce above $\sim 10 \mathrm{GHz}$. They disappear from mean profiles and we have captured very few single pulses in these phase windows at higher frequencies.

\subsection{Sporadic energy release in the emission zone}

Microbursts show that the radio emission region is neither uniform nor homogeneous. It must be highly variable in space and time, producing the short-lived bursts of radio emission from isolated regions within the emission zone. We therefore need some process which sporadically creates localized regions in which conditions are conducive to coherent radio emission. We don't know the radio emission mechanism, but nearly all models rely on a relativistic particle beam (as we discuss in Secs. 6] and Appendix A). The sporadic nature of the radio bursts tells us the driving beams are not steady. They must dissipate after their energy is given up to the radio emission, and be continually regenerated throughout the emission region.

The driving beams must themselves be driven by electric fields. In highly magnetized regions such as the pulsar magnetosphere, the driving $\mathbf{E}$ must be parallel to the local $\mathbf{B}$. Thus, $E_{\|}$must itself be sporadic: reaching high strengths, creating the particle beam, then - perhaps - being shorted out. This picture was originally suggested for the polar cap by Ruderman \& Sutherland (1975), and has been explored numerically by Levinson et al. (2005) and Timokhin \& Arons (2013). Their simulations verify that cyclic behavior can be a direct consequence of low-altitude magnetic pair production, as follows. Say we start with a finite $E_{\|}$, driven by the star's rotation, in a nearly empty gap region. It accelerates charges, which in turn create $\gamma$-rays by curvature radiation. The $\gamma$-ray photons decay into $e^{+} e^{-}$pairs, which fill the gap region and shield $E_{\|}$. Once the electric field is neutralized, particle acceleration ceases, the existing pairs stream out of the region, and the original $E_{\|}$is recovered as the gap again becomes (nearly) empty.

The radio microbursts tell us that a similar process is needed in the high-altitude radio emission regions of the Crab pulsar. However, the magnetic field is too low in those 

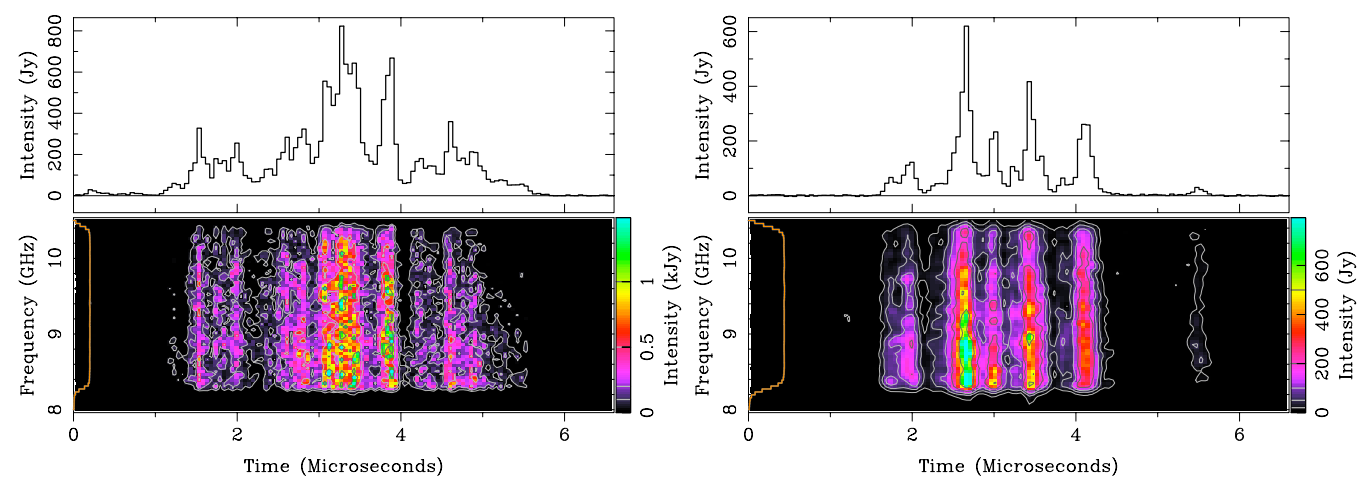

Figure 2. Two typical examples of Main Pulses. Upper panels show intensity integrated across our observing band. The radio emission comes in distinct bursts, each lasting less than a microsecond. Lower panels show the dynamic spectrum within our observing band; the orange line on the left shows the equalized frequency response of the receiver. The spectrum of each microburst is relatively broadband, spanning the full observing bandwidth (but compare the pulse shown in Fig. 3 where individual, narrow-band shots within the pulse can be resolved). Data were obtained with time resolution equal to the inverse of the observing bandwidth. For display purposes, both pulses have been smoothed to time resolution 51.2 ns and spectral resolution 78

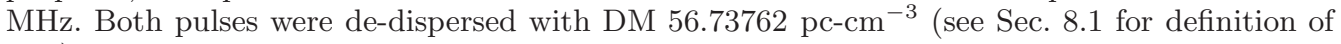
$\mathrm{DM})$.

regions for magnetic pair production to take place in situ. It may be that the particle beams which drive the radio emission have propagated from their natal regions above the polar caps, but it is not obvious that they would retain their identity and still have the sporadic and inhomogeneous nature required to explain the microbursts. Alternatively, two-photon pair creation may take place within the high-altitude gaps (e.g, Ho 1989, Cheng et al. 2000). The seed $\gamma$-rays are still thought to come from curvature radiation of charges accelerated by unshielded $E_{\|}$fields. The target photons (likely X-rays) may be thermal emission from the star's surface, or secondary photons from a low-altitude pair cascade. While this process has not been studied in detail, it seems likely that an oscillatory $E_{\|}$cycle can also be characteristic of high-altitude gap regions.

\subsection{Nanoshots}

Once in awhile a Main Pulse or a Low-Frequency Interpulse can be resolved into very brief nanoshots. We show examples in Fig. 3, others can be seen in Hankins et al. (2016). Because we believe these nanoshots provide a critical test of the radio emission mechanism, we highlight their properties here.

Timescales. Some of the nanoshots we have captured are unresolved at our best time resolution (a fraction of a nanosecond; the inverse of our observing bandwidth). Others are marginally resolved, lasting on the order of a nanosecond. The example pulse we show in Fig. 3 is substantially smoothed, for clarity of presentation. Nanoshots displayed at higher time resolution can be found in Hankins et al. (2003) and Hankins \& Eilek (2007).

Spectrum. Each isolated nanoshot we have captured is relatively narrowband, with fractional bandwidth $\delta \nu / \nu$ on the order of $10 \%$. Fig. 3 shows that the center frequency of the nanoshots is not fixed, but can be anywhere within our observing band. The product of the center frequency, $\nu_{\mathrm{obs}}$, and duration, $\delta t$, of a nanoshot turns out to be a useful diagnostic for the models. For each isolated nanoshot we have captured between 5 and $10 \mathrm{GHz}$ this product $\nu_{\mathrm{obs}} \delta t \sim O(10)$. 

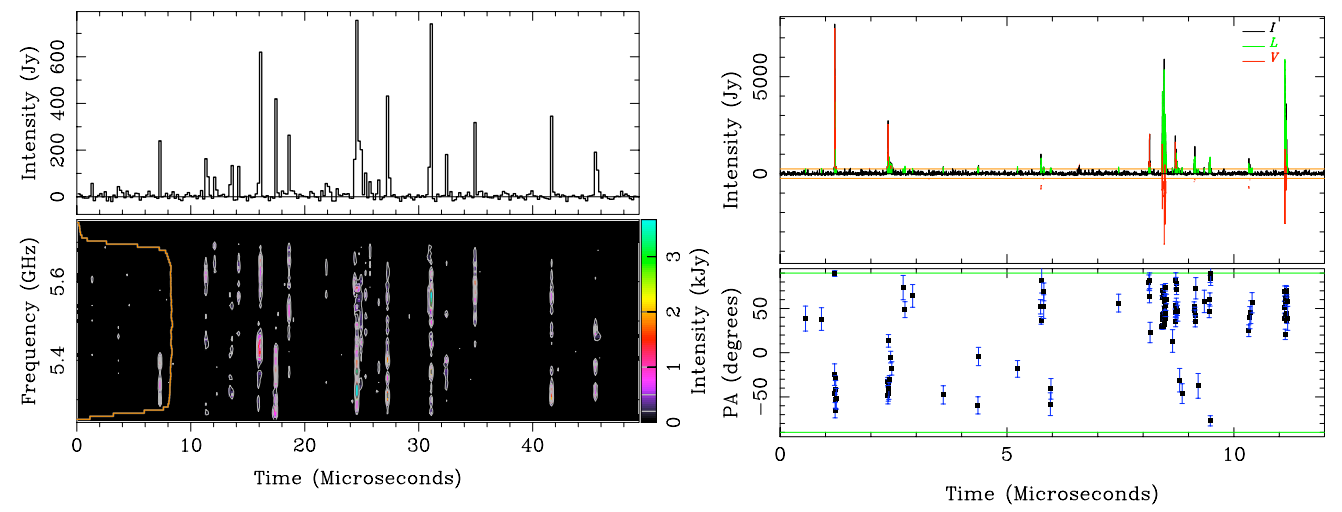

Figure 3. An example of nanoshots in a Main Pulse. Left figure shows the total intensity and

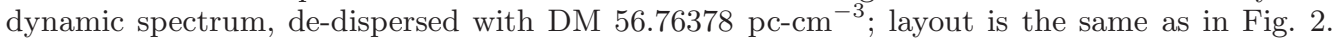
The radio emission in this pulse is confined to a set of well-separated nanoshots. Each nanoshot lasts on the order of a nanosecond, and is relatively narrowband (frequency spread $\delta \nu \sim 0.1 \nu$ ). Shown with time resolution $51.2 \mathrm{~ns}$ and spectral resolution $39.1 \mathrm{MHz}$. The right figure is a zoom into the nanoshots seen between 18 and $27 \mu$ s in the left figure, now displayed at $4 \mathrm{~ns}$ time resolution. At this resolution, individual nanoshots within the clump at $24 \mu$ s are now resolved. The different time smoothing in this view gives different peak fluxes for each nanoshot. The upper panel shows the total intensity (I; black), linearly polarized intensity (L; green) and circularly polarized intensity $(\mathrm{V}$; red). The lower panel shows the position angle of the linear polarization. Only polarized flux above 4 times the off-pulse noise (indicated by the orange lines in the top panel) is shown. Note the strong circular polarization of individual nanoshots can be of either sign.

Polarization. As the example in Fig. 3 shows, individual nanoshots tend to be elliptically polarized. While most of them show some linear polarization, they are often dominated by circular polarization $(\mathrm{CP})$. The sign of the $\mathrm{CP}$ can change from one nanoshot to the next, apparently at random.

Although microbursts in most Main Pulses and Low-Frequency Interpulses last longer and have a broader dynamic spectrum than do the nanoshots, we suspect that microbursts are incoherent superpositions of short-lived, narrow-band, nanoshots. In other words, all microbursts are "clumps of nanoshots". This is supported by the observationally motivated amplitude-modulated-noise model of pulsar emission (Rickett 1975). Because each such microburst is broadband (Sec. 5.1), the center frequencies of the nanoshots within a microburst must span a wide frequency range.

\section{Nanoshots as tests of radio emission models}

It has been hard, historically, to compare radio emission models to the data. The models address physical processes on plasma microscales, but most of the data can only address larger-scale, system-wide processes. We believe the nanoshots in the Main Pulse and Low-Frequency Interpulse, revealed by our very high time resolution observations, provide a key clue to the underlying physics.

In this section we use our data to confront three plausible models from Appendix $\mathrm{A}$ Because each model can be connected to the spectrum, duration and/or polarization of an individual nanoshot, we can constrain physical conditions which must hold in the radio emission region in order for that model to work.

For easy reference in the following discussion we refer back to the basic magnetospheric 


\begin{tabular}{|c|c|c|c|c|}
\hline Model & Secs. & Spectrum & Timescales & Polarization \\
\hline Strong plasma turbulence (SPT) & $6.1 \mathrm{A.1}$ & consistent & consistent & needs work ${ }^{a}$ \\
\hline Free electron masers (FEM) & $6.2 \mathrm{~A} .2$ & plausible & consistent & needs work $^{a}$ \\
\hline Cyclotron instability emission (CIE) & $6.3 \mathrm{~A} .3$ & challenged $^{b}$ & needs work ${ }^{c}$ & consistent \\
\hline
\end{tabular}

TABle 3. Overview of nanoshot models discussed in the text. ${ }^{a}$ Both SPT and FEM, as currently formulated, predict fully linear polarization, which disagrees with the observations. ${ }^{b}$ The densities needed for CIE to match observations are much higher than magnetosphere theories predict. ${ }^{c}$ The current formulation of CIE cannot address the short-lived nanoshots we observe.

parameters of the Crab pulsar in Table 1. We highlight the three models in Table 3 and summarize the plasma conditions required by each model in Table 4.

\subsection{Strong plasma turbulence}

When electrostatic turbulence is driven to large amplitude (which we call "strong plasma turbulence", SPT), a modulational instability creates localized Langmuir-wave solitons. These solitons are themselves unstable, generating electromagnetic waves which can escape the plasma, and potentially be observed as nanoshots. We generally follow Weatherall (1997, 1998); see also Sec. A.1 of the Appendix.

Spectrum. Individual bursts are emitted around the plasma frequency in the comoving plasma frame. If the plasma is moving out from the pulsar at a streaming speed $\gamma_{\mathrm{s}}$, we observe nanoshots centered at

$$
\nu_{\mathrm{obs}}^{\mathrm{SPT}} \sim 2 \gamma_{\mathrm{s}}^{1 / 2} \nu_{\mathrm{p}}
$$

where $\nu_{\mathrm{p}}$ is the plasma frequency, and everything is written in the observer's frame. Conditions required to put $\nu_{\mathrm{obs}}$ in the radio band are given in Table 4 and Sec. 6.4. Simulations by Weatherall (1998) predict the nanoshots will be relatively narrowband, with $\delta \nu / \nu_{\text {obs }} \sim \Lambda$, where $\Lambda$ measures the strength of the turbulence. Using $\Lambda \gtrsim 0.1$, from Weatherall $(1997,1998)$, this prediction agrees with our observations of isolated nanoshots (Sec. 5.3).

Timescales. The dynamic timescale for soliton collapse, and the duration of the consequent flare of radiation, is $\delta t \sim 1 /\left(2 \gamma_{\mathrm{s}}^{1 / 2} \Lambda \nu_{\mathrm{p}}\right)$, again written in the observer's frame. Using equation (6.1), this model predicts that radiation bursts observed at a few $\mathrm{GHz}$ last on the order of a nanosecond, and have the product $\nu_{\mathrm{obs}} \delta t \sim 1 / \Lambda$. These predictions are also consistent with our observations of isolated nanoshots.

Polarization. The collapsing soliton generates electromagnetic waves whose polarization is set by local plasma conditions. Weatherall (1998) worked in the context of a strongly magnetized plasma, in which charges can only move along the magnetic field. In this limit, emergent radiation around the plasma frequency is linearly polarized along the magnetic field. Because nanoshots from the Crab pulsar can show strong circular polarization, they cannot be explained by Weatherall's model as it stands. That model would have to be extended, perhaps to a plasma in which the natural modes are elliptically polarized.

\subsection{Free-electron-maser emission}

A relativistic electron beam, moving parallel to the magnetic field, generates Langmuir turbulence as usual. The interaction of the beam particles with that turbulence leads to coherent bunching of the beam charges, and consequent strong bursts of radiation. This 
model is similar to the SPT model, but here the beam itself is what radiates; emission from the background plasma is not considered. See also Sec. A.2 of the Appendix.

Spectrum. Bunched electron beams, moving at $\gamma_{\mathrm{b}}$, scatter on intense, localized electrostatic waves of the plasma turbulence. The scattered radiation can be treated as inverse Compton scattering of the Langmuir photons. If the plasma is at rest, the scattered photon frequency i.

$$
\nu_{\mathrm{obs}}^{\mathrm{FEM}} \sim 2 \gamma_{\mathrm{b}}^{2} \nu_{\mathrm{p}}
$$

(e.g. Benford 1992). Conditions required to put $\nu_{\mathrm{obs}}$ in the radio band are given in Table 4 and Sec. 6.4.

Timescales. Short-lived radiation bursts arise naturally in a free-electron maser, when the beam particles interact with electric fields in the Langmuir turbulence they have generated. Both the coherent charge bunching, and the consequent radiation bursts, are characterized by the plasma timescale for the background plasma: $\delta t \sim 1 / \nu_{\mathrm{p}}$ (e.g., Schopper et al. 2003). Thus, this model predicts the product $\nu_{\mathrm{obs}} \delta t \sim \gamma_{\mathrm{b}}^{2}$. Clearly the beam cannot be too fast; $\gamma_{\mathrm{b}}^{2} \sim O(10)$ is needed to match the frequency-duration product of our observed nanoshots.

Polarization. Both the oscillating electric field in the induced Langmuir turbulence, and the motion of beam charges in that field, are parallel to the local magnetic field. It follows that radiation bursts are linearly polarized, also parallel to B (e.g., Windsor \& Kellogg 1974). This model would also have to be extended to accommodate circular polarization. Perhaps the bunched beam charges have finite and synchronized pitch angles. This can work in the lab; Benford \& Tzach (2000) reported coherent synchrotron emission from bunched electrons in an incoming rotating beam. However, it is not clear that such a situation would arise naturally in a pulsar magnetosphere.

\subsection{Cyclotron instability emission}

A relativistic particle beam moving into a magnetized plasma generates transverse waves when it couples to the plasma through the anomalous cyclotron resonance. Because the waves can escape the plasma without mode conversion, this is a direct emission process. This theory has been applied to pulsars by Kazbegi et al. (1991, "K91") and Lyutikov et al. (1999, "L99", and references therein). To compare this model to Main Pulse nanoshots, we follow the specific problem setup assumed by those authors: onedimensional motion of a cold pair plasma, streaming outward from the pulsar at $\gamma_{\mathrm{s}}$, as well as a faster "primary beam" moving through that plasma at $\gamma_{\mathrm{b}}$. See also Sec. A.3 of the Appendix.

Spectrum. Emission proceeds through the first harmonic of the anomalous cyclotron resonance. The escaping radiation is around the resonant frequency:

$$
\nu_{\mathrm{obs}}^{\mathrm{CIE}}=\nu_{\mathrm{B}} \frac{4 \nu_{\mathrm{B}}^{2}}{\nu_{\mathrm{p}}^{2}} \frac{\gamma_{\mathrm{s}}^{3}}{\gamma_{\mathrm{res}}}
$$

where $\nu_{\mathrm{B}}$ is the electron cyclotron frequency and $\gamma_{\mathrm{res}}$ is the Lorentz factor of the resonant beam particles $\left(\gamma_{\mathrm{s}}<\gamma_{\text {res }}<\gamma_{\mathrm{b}}\right)$. All terms are written in the observer's frame, and the solution describes waves propagating nearly along the magnetic field. Because this is a resonant process, operating at the lowest harmonic, we guess the emission is relatively narrow-band. Conditions required to put $\nu_{\mathrm{obs}}$ in the radio band are given in Table 4 and Sec. 6.4

$\dagger$ If plasma is itself streaming at $\gamma_{\mathrm{s}}$, the boosted radiation is seen in the observer's frame as $\nu_{\mathrm{obs}}^{\mathrm{FEM}} \simeq \gamma_{\mathrm{b}}^{2} \gamma_{\mathrm{s}}^{-3 / 2} \nu_{\mathrm{p}}$, which adds another free parameter to the model. 
Timescales. Because this model has not been carried past the linear instability calculation, it cannot address short-lived nanoshots. One would expect the instability to generate resonant plasma waves, some remaining in the plasma to generate pitch-angle scattering, others potentially escaping to be seen as radio emission. Lyutikov considered possible saturation mechanisms (e.g. L99 \& references therein), but did not discuss selfgenerated coherent particle bunching, which would be needed for bright radio nanoshots.

Polarization. If the distribution functions of the electrons and positrons in a pair plasma are different, the normal modes of the plasma become circularly polarized for propagation close to the magnetic field (Allen \& Melrose 1982, K91). The CIE models of K91/L99 take advantage of this fact by assuming relative streaming between the two species. If the electrons are moving faster than the positrons, their model predicts lefthanded $\mathrm{CP}$; if the positrons are moving faster, one gets right-handed $\mathrm{CP}$.

\subsection{Nanoshots: implications for the emission region}

In this section we have compared three beam-driven radio emission models to our nanoshot data. Each model has it own requirements on the plasma density in the emission region, which we summarize here and in Table 4 . We also point out that each model requires further development before it can match all of the observations.

\subsubsection{SPT and FEM models}

Both of these models involve plasma turbulence driven by a relativistic particle beam, presumably via a two-stream instability. The SPT model emphasizes radio emission from collapsing solitons when the turbulence has become strong. The FEM model emphasizes radio emission from coherent charge bunching caused by the beam responding to the turbulence it has created.

In both models the emission frequency is related to the plasma frequency in the background plasma: one boosted by $\gamma_{\mathrm{s}}$, the other by $\gamma_{\mathrm{b}}^{4}$. In practice, of course, we expect both phenomena to contribute to the nanoshots. The relative importance of the two effects depends on the relative densities of the driving beam and the background plasma, neither of which we attempt to estimate.

To radiate between $100 \mathrm{MHz}$ and $10 \mathrm{GHz}$, the emitting plasma density and streaming or beam speeds must satisfy

$$
n \gamma_{\mathrm{s}} \sim\left(3 \times 10^{7}-3 \times 10^{11}\right) \mathrm{cm}^{-3} \quad \text { or } n \gamma_{\mathrm{b}}^{4} \sim\left(3 \times 10^{7}-3 \times 10^{11}\right) \mathrm{cm}^{-3}
$$

for SPT or FEM, respectively. Recalling that $\gamma_{\mathrm{b}}^{2} \sim O(10)$ is needed to match the frequency-duration product of the nanoshots, and that $\gamma_{\mathrm{s}}$ may be as low as $\sim 10^{2}$ in some models, we see that these two constraints are not dissimilar.

Comparing these constraints to the GJ density in the upper magnetosphere (Table 11), we see that the density enhancement, $\lambda=n / n_{\mathrm{GJ}}$, must be in the range given by $10^{2} \lesssim \lambda \gamma_{\mathrm{s}} \lesssim 10^{5}$ and/or $10^{2} \lesssim \lambda \gamma_{\mathrm{b}}^{4} \lesssim 10^{5}$ in the emitting region. For modest values of $\gamma_{\mathrm{s}}$ and $\gamma_{\mathrm{b}}$, the upper part of the required density (or $\lambda$ ) range is generally consistent with models of pair production close to the polar cap (as in Sec. 2.11). Perhaps pair cascades operating in high altitude gaps result in similar enhancements and streaming speeds.

The lower end of the density range is problematic, however. Previous authors (e.g. Kunzl et al. 1998, Melrose \& Gedalin 1999) have argued that models based on relativistic plasma emission have difficulty explaining radio emission from young pulsars. If the radio emission comes from low altitudes - over the polar cap - the GJ density there is too high, even with $\lambda \sim 1$, to be compatible with low radio frequencies. This problem is mitigated if the radio emission comes from higher altitudes, as it does in the Crab pulsar. Perhaps 
the lowest radio frequencies come from high altitude regions where the plasma density is not significantly enhanced over the GJ density.

\subsubsection{CIE model}

This model is also beam-driven, but the emission proceeds through the cyclotron resonance, which becomes unstable in the low magnetic fields found at high altitudes (for the specific model of K91/L99). Because this model includes relative streaming between electrons and positrons, it can explain the circular polarization we observe in the nanoshots.

Stringent conditions, however are required if this model is to explain the radio data. Matching the resonant frequency (equation 6.3) to the radio band requires

$$
\left(n \gamma_{\text {res }} / B^{3} \gamma_{\mathrm{s}}^{2}\right) \sim\left(1 \times 10^{40}-1 \times 10^{42}\right) \mathrm{cm}^{-3} \mathrm{G}^{-3}
$$

Even taking the most optimistic parameter choices within the standard model $\left(B \sim 10^{6}\right.$ $\mathrm{G}, \gamma_{\mathrm{res}} \sim 10^{6}$ and $\gamma_{\mathrm{s}}$ of order unity), we still need $n \sim 10^{16}-10^{18} \mathrm{~cm}^{-3}$ in order for this model to produce radio emission.

Such high densities require huge pair enhancements, $\lambda \sim 10^{9}-10^{11}$, much higher than predicted by models of polar-cap pair cascades (Sec. 2.1). For comparison, models of the Crab Nebula suggest the mean outflow density from the pulsar exceeds the GJ value by 5 or 6 orders of magnitude (e.g. Bucciantini et al. 2010). While that range also exceeds the $\lambda$ values predicted by pair cascade models, the density enhancement required if the CIE model is to match observations is higher still. Perhaps some process, such as magnetic reconnection, can dump large amounts of mass into high-altitude emission regions. Perhaps unusually low magnetic fields - which would lower the necessary density range - exist in such regions.

\subsubsection{What's missing?}

None of the three models, as currently formulated, can address all of the data. The SPT and FEM models can explain the existence of the nanoshots, but not their polarization. The CIE model can explain the circularly polarized emission, but cannot explain the existence of the nanoshots.

We suspect the way forward is a combination of the two approaches. If the electrons and positrons have different distributions - for instance, different streaming speeds the fundamental modes of the plasma are circularly polarized. Alternating signs of CP in different nanoshots require alternating dominance of one or the other species in each nanoshot. Perhaps that can exist in a highly turbulent pair cascade zone. Nanosecondlong bursts of coherent radio emission suggest that the emitting charges are coherently bunched. Perhaps that can come from the nonlinear evolution of one or both of these models, operating in a plasma which can carry circular polarization.

\section{The High-Frequency Interpulse: a different picture}

When we first studied individual High-Frequency Interpulses, in 2004 through 2006, we were astonished to find they have very different properties from Main Pulses and Low-Frequency Interpulses. Since then we have continued to study them, over several years, going to other telescopes and higher radio frequencies. The result is unchanged: High-Frequency Interpulses are very different from Main Pulses and Low-Frequency Interpulses, in ways no current model of pulsar radio emission can explain.

\subsection{Microbursts but no nanoshots}

The temporal signature of High-Frequency Interpulses is similar to that of Main Pulses and Low-Frequency Interpulses (Sec. 5), but with important differences. Single High- 


\begin{tabular}{ccc}
\hline Model & Conditions & Secs. \\
Strong plasma turbulence (SPT) & $n \gamma_{\mathrm{s}} \sim\left(3 \times 10^{7}-3 \times 10^{11}\right) \mathrm{cm}^{-3}$ & 6.1 6.4 A.1 \\
Free electron masers (FEM) & $n \gamma_{\mathrm{b}}^{4} \sim\left(3 \times 10^{7}-3 \times 10^{11}\right) \mathrm{cm}^{-3}$ & 6.2 6.4 A.2 \\
Cyclotron instability emission (CIE) & $n \gamma_{\mathrm{res}} \sim\left(1 \times 10^{22}-1 \times 10^{24}\right) B_{6}^{3} \gamma_{\mathrm{s}}^{3} \mathrm{~cm}^{-3}$ 6.3 6.4 A.3
\end{tabular}

TABle 4. Plasma conditions required for radio emission models discussed in Sec. 6 to match the spectrum of the nanoshots. The density of the pair plasma is $n$; for comparison, Table 1 shows the GJ density $n_{\mathrm{GJ}} \sim\left(2 \times 10^{6}-2 \times 10^{7}\right) \mathrm{cm}^{-3}$ in the upper magnetosphere. The Lorentz factor $\gamma_{\mathrm{s}}$ describes the streaming speed of the pair plasma; $\gamma_{\mathrm{b}}$ describes the speed of the particle beam that drives FEM emission, which must satisfy $\gamma_{\mathrm{b}}^{2} \sim O(10)$ to match the observations; $\gamma_{\text {res }}$ describes energy of the beam particles which participate in the cyclotron resonance. The magnetic field is scaled to $10^{6} \mathrm{G}$, the smallest field likely within the standard picture of the magnetosphere.

Frequency Interpulses typically contain two or three microbursts, each lasting a few microseconds and overlapping in time. Fig. 4 shows two examples. As with Main Pulses and Low-Frequency Interpulses, individual High-Frequency Interpulses are much shorter-lived than the corresponding component in the pulsar's mean profile. Single pulses can occur anywhere within the mean-profile envelope; more than one pulse, typically separated by a few hundred $\mu$ s, can occur in the same rotation period.

Also as with the Main Pulse and Low-Frequency Interpulse, the emission region for the High-Frequency Interpulse is dynamic. Localized hot spots, with conditions favorable to radio emission, are created intermittently throughout the broad emission region. Each hot spot emits a burst of coherent radio emission, then dies away, in only a few $\mu$ s. Similar hot spots are regenerated again and again throughout the extended emission region that is the source of the High-Frequency Interpulse.

There are, however, some differences between the time signature of High-Frequency Interpulses, and that of Main Pulses and the Low-Frequency Interpulses. Each microburst in a High-Frequency Interpulse lasts about ten times longer than that in a Main Pulse (compare Figs. 2 and 4). We have never recorded a High-Frequency Interpulse with the well-separated, sub- $\mu$ s bursts that characterize the Main Pulses, nor have we ever captured nanoshots in a High-Frequency Interpulse. These details suggest to us that the energy storage/release mechanism - be it particle beams driven by $E_{\|}$, or something completely different - is not the same in the two types of emission regions.

\subsection{Spectral emission bands}

The unusual character of High-Frequency Interpulses is clearest from their spectra. To quote Zheleznyakov et al. (2012), the spectra revealed by our observations are "completely unexpected against a backdrop of forty-year-long studies of pulsar radio emission".

Comparing the lower panels of Figs. 2] and 4 illustrates the difference. Where the burst spectrum of a Main Pulse or Low-Frequency Interpulse is continuous across our observing band, the spectrum of a High-Frequency Interpulse is characterized by spectral emission bands. Each burst in a High-Frequency Interpulse contains its own band sets. For pulses with more than one burst and band set, the bands associated with second or third bursts tend to be at slightly higher frequencies than those in the first burst (Fig. 4 shows good examples).

The spacing between adjacent bands is not constant, as one might expect for simple harmonic emission. Instead, the band spacing increases with frequency, while keeping the 

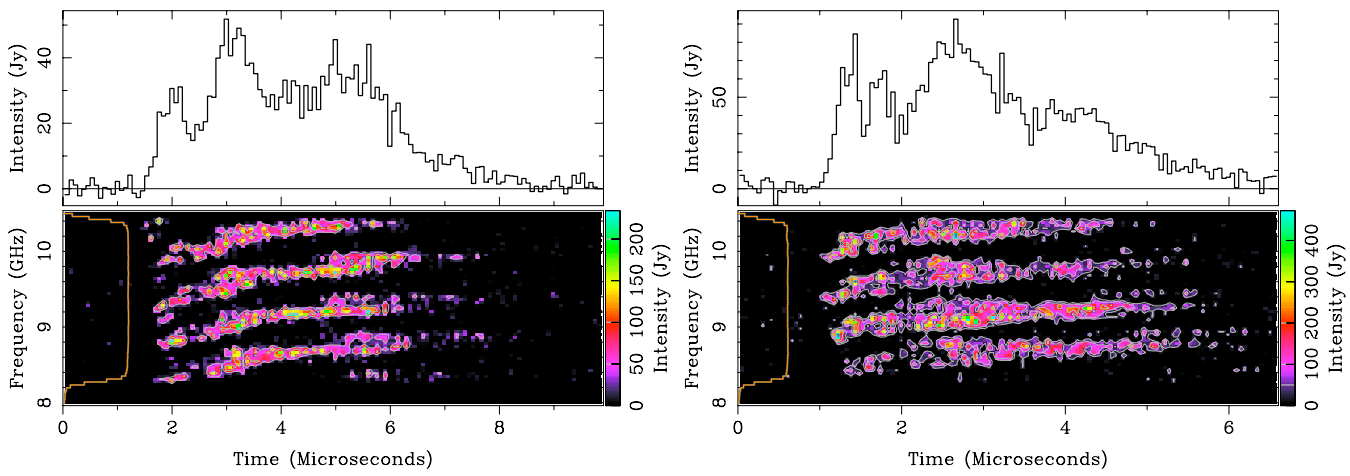

Figure 4. Two typical examples of High-Frequency Interpulses, shown with the same layout as in Fig. 2 Each pulse contains a small number of bursts, each one lasting a few microseconds. Comparison to Fig. 2 shows that bursts in this component are fewer, and longer-lived, than in a typical Main Pulse. The dynamic spectra show the radio emission is concentrated in narrow spectral emission bands. These bands are very different from the Main Pulse spectra (Figs. 2 and 3), and were not predicted by any model of pulsar radio emission. Each microburst in a High-Frequency Interpulse has its own band sets: four sets can be seen in the pulse on the left, and (probably) three can be found in the pulse on the right. Pulse on left was de-dispersed with

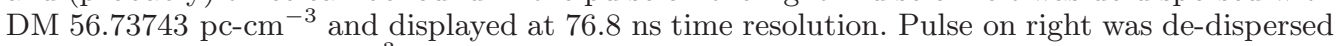
with DM $56.75017{\mathrm{pc}-\mathrm{cm}^{-3}}^{-3}$ and displayed with time resolution $51.2 \mathrm{~ns}$. Both pulses displayed with $78.125 \mathrm{MHz}$ spectral resolution.

fractional separation constant. Our data between 6 and $30 \mathrm{GHz}$ are well fit by $\Delta \nu \simeq 0.06 \nu$ (details in Hankins \& Eilek 2007, Hankins et al. 2016). Interestingly, our formal fit to the band spacing is consistent with zero band spacing at zero frequency, even though we have seen no High-Frequency Interpulses below $5 \mathrm{GHz}$.

We have never seen a High-Frequency Interpulse which did not have emission bands across our full observing band. Thus, although we have never been able to observe the full 5-30 $\mathrm{GHz}$ bandwidth at one time, we think it likely that each burst of a HighFrequency Interpulse contains at least 30 emission bands, proportionally spaced from 5 $\mathrm{GHz}$ upwards.

\subsection{Models for spectral emission bands}

The High-Frequency Interpulse of the Crab pulsar is the only place where ordered spectral emission bands have been seen in a pulsar. The bands cannot be satisfactorily explained by any current model of pulsar radio emission (including those listed in Appendix $\mathrm{A}$ ).

To search for new models, we note the resemblance between emission bands in the High-Frequency Interpulse and "zebra bands" seen in dynamic spectra of type IV solar flares. Zebra band sets can have from a few up to $\sim 30$ spectral bands; they also have band spacing that increases with frequency (e.g. Chernov et al. 2005). Two classes of models have been proposed for zebra bands: geometric effects and resonant plasma emission. Perhaps of these models will help us understand the emission bands in the Crab pulsar.

\subsubsection{Geometric models}

The striking regularity of the bands suggests an interference or propagation effect. For instance, if some mechanism splits the emission beam coherently, it may interfere with itself, creating emission bands. Alternatively, perhaps cavities form in the plasma and trap some of the incoming radiation, imposing a discrete frequency structure on the escaping signal. Both possibilities have been suggested for solar zebra bands (e.g. Ledenev 
et al. 2001; LaBelle et al. 2003). The necessary plasma structures must be small, on the order of a few to a few hundred centimeters.

While we find this idea attractive, making it work is challenging. One issue is the incoming radiation. If the bands are due to a geometrical effect, the incoming radiation must be coherent over the full 5-30 GHz band. The narrow-band shots which characterize Main Pulse and Low-Frequency Interpulse emission do not work. A quite different emission mechanism is needed - perhaps from a double layer. Charges accelerated in the potential drop of a double layer emit broadband radiation (Kuijpers 1990). If the double layer thickness is $\sim c / 2 \pi \nu_{\mathrm{p}}$ (following Carlqvist 1982 for a relativistic lepton double layer), the radiation bandwidth may be sufficiently large.

The more difficult challenge, however, is the basic geometry. What long-lived plasma structures can create the necessary interference or wave trapping? How can those structures be created or maintained so consistently, within a dynamic magnetosphere, to give a steady $\Delta \nu / \nu$ which has not changed over the seven years we have studied this star? We have not come up with a good solution to this problem; new ideas are needed.

\subsubsection{Double plasma resonance}

This model was also proposed to explain solar zebra bands (e.g., Kuijpers 1975, Zhelezniakov \& Zlotnik 1975, Winglee \& Dulk 1986). Recent radio observations of solar flares (Chen et al. 2011) are in good agreement with the model. If conditions are right (as detailed in Appendix B), resonant emission occurs when the plasma frequency is an integer multiple, $s$, of the electron gyrofrequency:

$$
\nu_{\mathrm{obs}}^{\mathrm{DPR}} \simeq \nu_{\mathrm{p}} \simeq s \nu_{\mathrm{B}}
$$

If the upper-hybrid turbulence excited by the instability converts to electromagnetic modes which can escape the plasma, we will see emission bands at discrete values of $\nu_{\text {obs. }}$. In a non-uniform plasma the resonance for each integer $s$ occurs at different spatial locations; the band spacing depends on the specific structure of the plasma density and magnetic field. Zheleznyakov et al. (2012) suggested this mechanism can also account for the emission bands in the High-Frequency Interpulse. While we also find this model promising, two problems are apparent.

One problem is the plasma setting. The double plasma resonance requires superthermal particles moving across a weak magnetic field (with $\nu_{\mathrm{B}} \ll \nu_{\mathrm{p}}$ ). This situation arises naturally in the magnetic bottles created by solar coronal loops, from which only particles with low pitch angles can escape. However, such geometry is not part of our standard picture for the pulsar magnetosphere, where particle beams moving along the magnetic field are thought to be common (e.g., Secs. 30r 5.2). Perhaps magnetic traps can form in dynamic regions of the upper magnetosphere, or associated with neutral current sheets (e.g. Zheleznyakov et al. 2012).

A second problem is the numbers. To put the resonant frequencies in the radio band, the plasma density must be high and the magnetic field extremely low. To get $\nu_{\text {obs }} \sim 5-30$ $\mathrm{GHz}$, the plasma must have $n \sim 10^{11}-10^{13} \mathrm{~cm}^{-3}$ and $B \sim 10^{2}-10^{4} \mathrm{G}$. Referring to Table 1 we see these parameter ranges do not easily fit anywhere in the standard picture of the magnetosphere. Higher densities and much lower fields are needed. Perhaps the necessary magnetic loops or current sheets, if they do exist in the upper magnetosphere, have the right parameters; but no models yet predict such structures. Once again, new ideas are needed. 


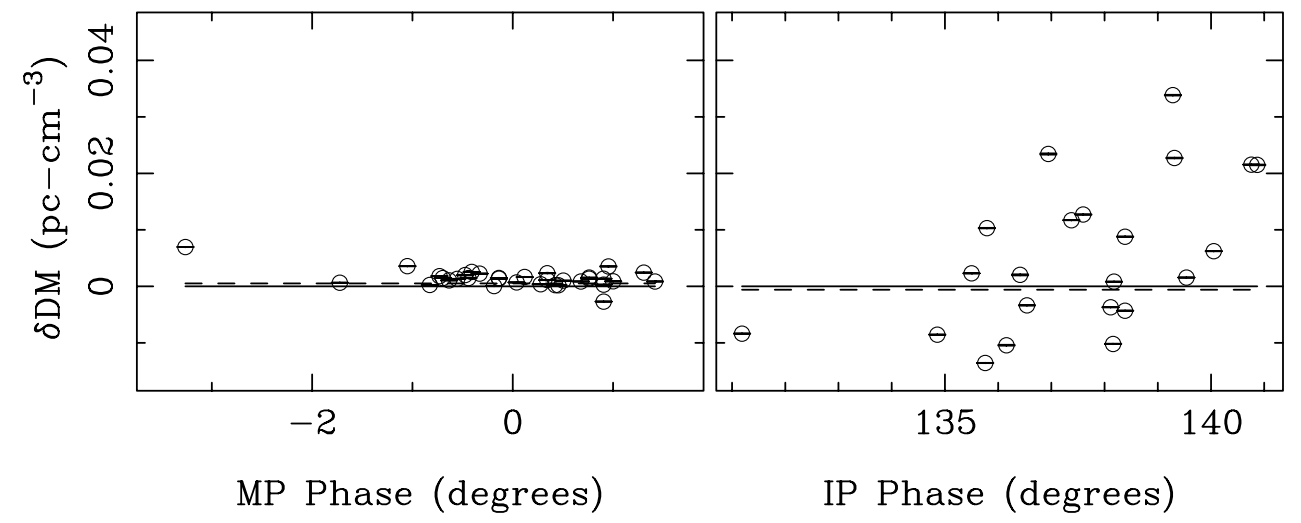

FIGURE 5. Excess dispersion measures, relative to Jodrell Bank monitoring, for 58 pulses captured within 80 minutes of each other during a typical observing day. Left panel shows $\delta(\mathrm{DM})$ values for Main Pulses; right panel shows $\delta(\mathrm{DM})$ for High-Frequency Interpulses. Observed at center frequency $6500 \mathrm{MHz}$, with bandwidth $1 \mathrm{GHz}$; plotted against the rotation phase at which each pulse arrived (compare the mean profiles in Fig. 1). See Hankins et al. (2016) for details of method. The solid lines show the Jodrell Bank value (corresponding to $\delta(\mathrm{DM})=0$ ); the dotted lines are the mean $\delta(\mathrm{DM})$ for each set of pulses. Dispersion of Main Pulses is approximately consistent with the Jodrell Bank value, but High-Frequency Interpulses have significant intrinsic dispersion $(\delta(\mathrm{DM}) \neq 0)$, with large pulse-to-pulse scatter.

\section{Emission region of the High-Frequency Interpulse}

The spectral emission bands are not the only unusual property of the High-Frequency Interpulse. Unlike Main Pulses and Low-Frequency Interpulses, High-Frequency Interpulses are partially dispersed within the magnetosphere before they leave the pulsar. In addition, their linear position angle shows no rotation across the entire mean-profile component. Both properties give us additional clues on the origin of High-Frequency Interpulses.

\subsection{Intrinsic dispersion in the High-Frequency Interpulse}

To quantify magnetospheric dispersion, we use the Dispersion Measure ("DM"), defined as follows. The signal propagation time from a cosmic source depends on the group velocity in the plasma through which it propagates: $t_{\mathrm{p}}(\nu) \propto(d \omega / d k)^{-1}$. For cold, weakly magnetized plasma (such as the interstellar medium), the dispersion relation is well known, and we have

$$
t_{\mathrm{p}}(\nu) \propto(\mathrm{DM}) \nu^{-2} \quad \text { where } \quad \mathrm{DM}=\int n d z
$$

Thus, the difference in pulse arrival times at two frequencies measures the column density between the signal source and us. This quantity is conventionally expressed as the DM, with units of pc-cm ${ }^{-3}\left(1 \mathrm{pc}-\mathrm{cm}^{-3} \simeq 3 \times 10^{18} \mathrm{~cm}^{-2}\right)$.

Nearly all of the dispersion for the Crab pulsar comes from the large plasma column between us and the pulsar - plasma in the Crab Nebula and the intervening interstellar medium. To focus on the small contribution from the pulsar itself, we express our results relative to the average DM which is tracked monthly by the Jodrell Bank Radio Observatory $\mathbb{H}$ This excess DM for an individual pulse, relative to the Jodrell Bank value, we call $\delta(\mathrm{DM})$.

$\dagger$ Determined from relative arrival times of bright components in mean profiles measured at 610 and $1400 \mathrm{MHz} ;$ http://www.jb.man.ac.uk/ pulsar/crab.html 
Fig. [5 shows $\delta(\mathrm{DM})$ values for Main Pulses and High-Frequency Interpulses on a typical observing day. We find $\delta(\mathrm{DM})$ for Main Pulses is approximately consistent with zero; the Jodrell Bank values describe the Main Pulse well. However, $\delta(\mathrm{DM})$ for High-Frequency Interpulses differs significantly from zero and fluctuates strongly from pulse to pulse. It

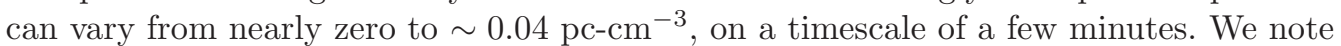
that observations of Main Pulses and High-Frequency Interpulses were interspersed with each other during a typical observing run. No intervening foreground "screen" could change rapidly enough, and just in phase with the pulsar's rotation, to exist only in front of the High-Frequency Interpulse. It is therefore clear that the excess DM of the High-Frequency Interpulse comes from the pulsar itself, not an intervening screen.

We find no evidence that $\delta(\mathrm{DM})$ depends on observing frequency between 5 and $30 \mathrm{GHz}$ where we have captured High-Frequency Interpulses. This suggests the magnetospheric dispersion law obeys $t_{\mathrm{p}}(\nu) \propto \nu^{-2}$ - although it need not be the cold plasma law assumed in equation (8.1). We note that a few $\delta(\mathrm{DM})$ values in Fig. 5 are negative. We do not interpret this as inverted dispersion behavior $\left(d t_{\mathrm{p}} / d \nu>0\right)$ in the pulsar. Rather, it seems likely that the Jodrell Bank value, which is based on mean profiles, contains a small contribution, the mean excess dispersion, $\langle\delta(\mathrm{DM})\rangle$, from the pulsar itself.

Interpreting our result in terms of plasma conditions in the emission region is difficult, because we do not know the true dispersion law in the region. Magnetospheric dispersion relations have been well studied only for low-altitude polar cap regions (e.g Arons \& Barnard 1986, Melrose \& Gedalin 1999). While the effective DM has not been calculated for these laws, we suspect the more complex physical situation in such strongly magnetized regions would cause more complex $t_{\mathrm{p}}(\nu)$ behavior than the simple form in equation (8.1).

As a possible example of dispersion in the upper magnetosphere, consider dispersion in a relativistic, weakly magnetized plasma. If $\langle\gamma\rangle$ measures the mean internal energy of the plasma, the DM over a path $L$ turns out to be $\simeq n L /\langle\gamma\rangle$ (following, e.g. Shcherbakov 2008). Guessing $L \sim 0.1 R_{\mathrm{LC}}$ for a high-altitude emission zone, we need $n /\langle\gamma\rangle \sim 4 \times 10^{9}$ $\mathrm{cm}^{-3}$ to account for the typical $\delta(\mathrm{DM}) \sim .02{\mathrm{pc}-\mathrm{cm}^{-3}}^{-3}$ we see in the High-Frequency Interpulse. Comparing this result to the high-altitude GJ densities (Table 1), we find a density enhancement $\lambda \sim\left(10^{2}-10^{3}\right)\langle\gamma\rangle$ would be needed. This is generally consistent with predictions of pair cascade models, as long as $\langle\gamma\rangle$ is not too large.

The more interesting clue, however, is the $\delta(\mathrm{DM})$ variability. The plasma column around or above the emission site fluctuates by $n /\langle\gamma\rangle \sim 4 \times 10^{9} \mathrm{~cm}^{-3}$, on timescales of no more than a few minutes. This shows the region creating the DM excess of the High-Frequency Interpulse is not some high-density region that happens to sit above the emission zone. It must be an intrinsic part of the dynamic emission zone.

\subsection{Linear polarization in the High-Frequency Interpulse}

High-Frequency Interpulses are linearly polarized. Figure 6 shows two typical pulses, both of which are strongly polarized with nearly constant position angles. The polarization provides two key diagnostics of the emission region for this component.

Such strong polarization - 90\% is typical - constrains the extent of the emission region. We argued in Sec. 2.3 that the phase coincidence of the High-Frequency Interpulse and the high-energy pulse P2 suggests the two features originate in similar parts of the magnetosphere. However, if the High-Frequency Interpulse comes from a wide range of altitudes - say throughout a slot gap or an outer gap - it would be depolarized by caustic effects (Dyks et al. 2004). The strong polarization of the High-Frequency Interpulse tells us caustic depolarization is not important for this component. Therefore, its emission 

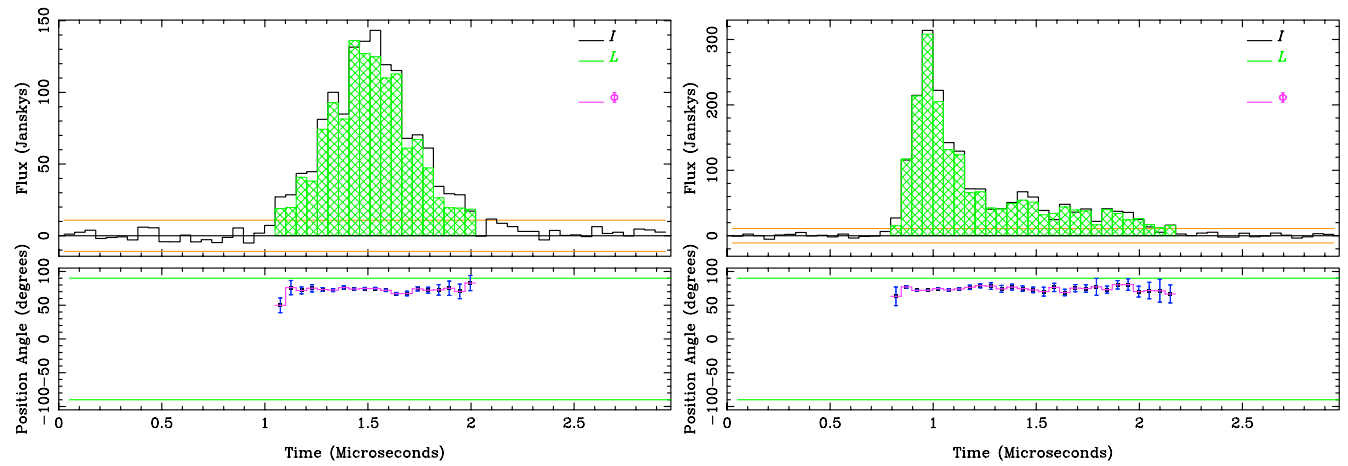

Figure 6. Polarization of two High-Frequency Interpulses, captured between 17.75 and 22.75 $\mathrm{GHz}$, within two minutes of each other on one observing day. De-dispersed with DM 56.79476 pc- $\mathrm{cm}^{-3}$ and displayed with $51.2 \mathrm{~ns}$ time resolution. The pulse on the left arrived close to the leading edge of the High-Frequency Interpulse component in the mean profile (Fig. 1); the pulse on the right arrived close to the trailing edge. For each pulse, the top panel shows the total flux (I; solid line) and linearly polarized flux (L; green filled area). The lower panel shows the position angle of the linear polarization $(\phi)$. Polarization is shown only for points above three times the off-pulse noise (indicated by the two orange lines in the top panel).

zone must occupy only a small fraction of the extended caustic region that is probably the source of pulsed high-energy emission.

Figure [6 also shows that the polarized position angle remains constant throughout a single pulse. This angle is independent of the rotation phase at which the pulse appears. For example, the two pulses in Figure 6 arrived at rotation phases separated by $6.5^{\circ}$ (which can be compared to the $9.0^{\circ}$ width of the mean-profile component; Hankins et al. 2015), yet both pulses show the same, steady polarization angle.

The position angle of pulsar radio emission reveals the direction of the magnetic field in the emission zone. Radio emission is polarized either parallel or perpendicular to the local field, depending on the emission model. The polarization angle observed at earth therefore depends on the direction of the field as projected on the sky plane. A change of angle through a pulse is interpreted as a change of magnetic field direction. The classic example is radiation from the low-altitude polar gap, which is a region with diverging field lines. As the star rotates past the line of sight, creating the radio pulse, the projected field direction rotates across the pulse (e.g. Radhakrishnan \& Cooke 1969).

The lack of such position-angle rotation in the High-Frequency Interpulse tells us the direction of the magnetic field is constant throughout its emission region. The field does not diverge within the region, as it does in the polar gap. Its direction does not change with time, as might be expected in a turbulent region dominated by plasma inertia. The emission region for the High-Frequency Interpulse must be inhomogeneous - in order to account for the variable dispersion - but must also contain an ordered magnetic field. Perhaps we are seeing a region filled with field-aligned density fluctuations.

\section{Summary and lessons learned}

In this paper we have reviewed our high-time-resolution observations of the Crab pulsar and compared them to various models for pulsar radio emission physics. We offer hearty congratulations to the reader who has made it this far. To summarize, our key results are as follows.

- The bright radio pulses come from high altitudes, not from the polar caps. Their 
emission regions are probably co-spatial with the gaps or current sheets responsible for high-energy emission. We use our data to probe physical conditions in the outer reaches of the magnetosphere.

- Variability of the radio signal on microsecond time scales, or longer, is generally consistent with the picture of cyclic pair cascades in an unshielded gap. The details, however, remain unclear; we suggest our radio data motivate new study of cascades in high-altitude emission regions.

- Three emission models - strong plasma turbulence, free-electron masers, and cyclotron instability emission - compare fairly well to the nanoshots which characterize the Main Pulse and the Low-Frequency Interpulse. However, none of them can explain all of the observations; further work is needed.

- The spectral emission bands which characterize the High-Frequency Interpulse confound every radio emission model yet proposed. The model which comes closest is doubleresonant emission, but it requires extreme conditions which push the envelope of current magnetosphere models.

- The variable dispersion and strong polarization of the High-Frequency Interpulse show the emission zone for that component is a localized, dynamic region that is probably threaded by a uniform magnetic field.

Our analysis makes it clear that we do not yet have all the answers about the pulsar's magnetosphere and how it makes coherent radio emission. We have, however, learned some useful lessons which should help us going forward.

- The Crab pulsar has at least one radio emission zone that is not predicted by current models.

Strong radio pulses from the Crab pulsar come from localized zones within extended high-energy emission regions - probably gaps or separatrices associated with each magnetic pole. We infer this from several facts. The Main Pulse and Low-Frequency Interpulse appear at the same rotation phases as the two strong components of the high-energy mean profile, and the viewing angle of the star does not allow a low-altitude polar cap origin for the latter. Furthermore, these two components have the same radio characteristics. This suggests they come from the same emission physics, which is consistent with their origin in two similar regions connected to the two magnetic poles.

The story is much less clear, however, for a third radio component. The High-Frequency Interpulse has very different characteristics from the other two. This suggests it comes from a region in which different emission physics is operating, but we do not know where that is. Because the High-Frequency and Low-Frequency Interpulses appear at similar rotation phases, we might think they come from different parts of one polar caustic. However, the most likely models suggest the High-Frequency Interpulse arises in a dense, turbulent region with uniform magnetic field direction. It is not clear where such a region exists within our current picture of the high-altitude magnetosphere.

- High-altitude emission zones are unsteady places. Their dynamical state is reflected in the radio emission.

Radio emission from the Crab pulsar is not steady. It comes in short-lived bursts which can occur anywhere within the probability envelope defined by the mean profile. We conclude from this that the mechanism which drives the coherent radio emission exists only sporadically throughout the emission zone. Whatever drives the driver must also be sporadic. The energy source builds up, is depleted to radio emission, and is soon regenerated to repeat the cycle.

If the driver is a relativistic particle beam - as is likely for the Main Pulse and LowFrequency Interpulse - the duty cycle reflects oscillatory behavior of parallel electric fields 
which drive the beams. This picture is generally consistent with the behavior expected for unsteady pair cascades in gap regions. If the Main Pulse and Low-Frequency Interpulse come from high-altitude caustic regions associated with the star's two magnetic poles, an unsteady "sparking" cycle must occur there, just as has been predicted for the lowaltitude polar cap.

The driver is less certain for the High-Frequency Interpulse, because its radio emission mechanism is less clear. Perhaps unsteady reconnection is taking place in the mysterious region which gives rise to this component.

- The radio emission regions are either denser, or more tenuous, than predicted by standard pair cascade models, but we do not know which.

Radio bursts in Main Pulses and Low-Frequency Interpulses are clumps of nanoshots: short-lived, narrow-band, polarized flashes of radio emission. No models yet proposed can fully explain the nanoshots, but some come close. One class of models involves strong plasma turbulence, either directly (via soliton collapse) or indirectly (via the bunched response of the driving beam). These models require fairly low densities within the emission zones - comparable to, or somewhat above, the GJ density. An alternative model invokes emission from a cyclotron instability. This model requires substantially higher densities in the emission zones, significantly above the GJ density for the region and also well above densities predicted by current pair cascade models. None of these models are the final word on the nanoshots, but all of them appear to disagree with predictions of the pair cascade models.

Two other arguments also suggest high densities. While the emission physics behind the High-Frequency Interpulse is not well understood, the promising double-resonant model of the spectral bands requires high densities in the relevant emission zone. In addition, some models of the Crab's pulsar wind nebula require high densities in the wind leaving the pulsar. Neither of these models is likely to be the final word on the High-Frequency Interpulse or the nebular wind, but both suggest densities higher than pair cascade models can produce. We conclude that the pair cascade - the only mechanism yet proposed to increase magnetospheric density over the fiducial GJ value - is not yet understood.

- Radio emission can be a powerful probe of magnetospheric conditions, but the models need more work.

We have discussed the radio emission models we find the most promising at present, and the limitations of each one. Models based on plasma turbulent emission have been extended to nonlinear regimes, and thus can address short-lived nanoshots. However, they are restricted to electrostatic turbulence in strongly magnetized regions, so they cannot account for circular polarization in the nanoshots. Models based on cyclotron emission have been constructed to admit circular polarization. However, they have not been developed past the linear regime, so they cannot address the behavior of the nanoshots. Models based on solar zebra bands are a promising approach to the spectral emission bands, but they require unusual physical situations which are not part of our standard picture of the magnetosphere.

Our opinion is that these models - which we have highlighted - can benefit by continued development, and help us truly understand conditions in the high-altitude radio emission zones. These are not the only options, however. Many other models, including those listed in Appendix $\mathrm{A}$ have promise but have not been developed to the point where they can be compared to data in any quantitative way. Perhaps one of them will turn out, in the end, to be a better answer for radio emission from the Crab pulsar.

We are grateful to Gregory Benford, Maxim Lyutikov and Don Melrose for entertaining discussions and thoughtful reviews that have helped our understanding and appreciation 
of pulsar emission physics. We particularly thank Glenn Jones and many of our students, including David Moffett, Jeff Kern, Jared Crossley, Tracey Delaney, Eric Plum, Joe Dickerson, and James Sheckard for their critical help at the telescopes during our observing sessions. The Arecibo Observatory is operated by SRI International under a cooperative agreement with the National Science Foundation, and in alliance with Ana G. Méndez-Universidad Metropolitana and the Universities Space Research Association. The Very Large Array and the Green Bank Telescope are run by the National Radio Astronomy Observatory, a facility of the National Science Foundation, operated under cooperative agreement by Associated Universities, Inc.

\section{Appendix A. Models proposed for pulsar radio emission}

A wide range of models have been proposed over the years to explain the mechanism by which pulsars make coherent radio emission. The models vary in both the plasma-scale processes responsible for the radiation and the magnetosphere conditions under which they can operate.

We do not attempt to give a full review of all possible radio emission models. The reader who has the strength can refer to the excellent reviews by Melrose (such as Melrose 1995) for a more complete discussion. Instead, we present overviews of several models which are commonly proposed and/or of particular interest for the Crab pulsar.

\section{A.1. Strong plasma turbulence}

This mechanism is known to operate in solar radio bursts, but must be revisited for the high magnetic fields and relativistic pair plasmas relevant to pulsars. An instability - most likely two-stream, driven by an energetic particle beam - drives Langmuir turbulence. That turbulence cannot escape the plasma directly; the Langmuir waves must undergo some nonlinear process which converts part or all of their energy into escaping radiation.

One possibility for this mode conversion involves modulational instabilities in strong Langmuir turbulence that drive the growth ("collapse") of localized knots of strong electric field, generally called solitons. Nonlinear wave-wave coupling within the solitons generates bursts of electromagnetic radiation - "nanoshots" - which can escape the plasma (e.g. Robinson 1997). Weatherall $(1997,1998)$ has studied this process in the pulsar context.

The duration and spectrum of the nanoshots provide key comparisons to the data. In Weatherall's simulations, the rate of collapse of the electrostatic wave packet and subsequent conversion to electromagnetic modes is on the order of $\sim \Lambda \nu_{\mathrm{p}}$, where $\nu_{\mathrm{p}}$ is the plasma frequency, and $\Lambda=E_{\mathrm{t}}^{2} / 8 \pi n m \bar{v}_{\|}^{2}$ measures the strength of the turbulence ( $E_{\mathrm{t}}$ is the turbulent wave electric field, and $\overline{v_{\|}}$is the velocity spread of the pair plasma). The timescale for soliton collapse, and the duration of the nanoshot produced by each collapse, is therefore $\delta t \sim 1 / \Lambda \nu_{\mathrm{p}}$. The spectrum of the nanoshot will be be relatively narrowband, $\delta \nu \sim 1 / \delta t \sim \Lambda \nu_{\mathrm{p}}$. Weatherall found that strong, localized solitons appear for $\Lambda \gtrsim 0.1$.

Weatherall's calculation assumes the radiative loss time scale is no longer than dynamic timescale for soliton collapse. This holds for packet sizes smaller than $\sim c / \Lambda \nu_{\mathrm{p}}$, which is consistent with other estimates of soliton size (e.g., Weatherall \& Benford 1991).

Polarization of the nanoshot is another key test. The collapsing soliton generates electromagnetic waves with polarization set by local plasma conditions. Weatherall (1998) worked in the context of a strongly magnetized plasma. In this limit, emergent radiation around the plasma frequency - such as the nanoshots - linearly polarized along the magnetic field (c.f. the O mode of Arons \& Barnard 1986). 
Because strong Langmuir turbulence is an incoherent superposition of localized solitons (e.g. Robinson 1997), the radiation "shot" produced by each soliton is independent of those from its neighbors. This agrees nicely with the amplitude-modulated noise model suggested by high-time-resolution observations of pulsar radio emission (Rickett 1975, also Sec. 5.31). We compare this model to the Crab pulsar in Sec. 6.1]

\section{A.2. Free-electron maser emission}

In this model, a relativistic beam encounters an electric field that varies in space and time. Charges interacting with the field bunch together in a resonant interaction, and emit intense, coherent bursts of radiation. This process has been extensively studied in the lab, where particle beams pass through externally applied electromagnetic fields ("wigglers") and radiate in a narrow cone beamed along the initial beam direction.

This interesting idea has not been taken very far in pulsar applications. Fung \& Kuijpers (2004) proposed a model in which a high- $\gamma$ beam propagates through a strong, transverse EM wiggler in a nearly unmagnetized background plasma. Their simulations found the expected collective particle bunching, resulting in short-lived, narrow-band bursts of radiation, on a timescale governed by the frequency of the imposed EM wiggler. However, neither an externally applied transverse wiggler, nor a nearly unmagnetized plasma, seem easy to maintain in a pulsar magnetosphere.

A more likely possibility is that the particle beam generates its own parallel wiggler. Baker et al. (1988), also Weatherall \& Benford (1991), suggested that when beam-driven Langmuir turbulence becomes strong, both it and the driving beam will become spatially inhomogneous. In that situation, the collective motion of charge bunches interacting with electrostatic fluctuations in the turbulence results in coherent, forward-beamed radiation (e.g. Kato et al. 1983, Schopper et al. 2003). For weak Langmuir turbulence, these models predict linearly polarized radiation at $\sim \gamma^{2} \nu_{\mathrm{p}}$, where $\nu_{\mathrm{p}}$ is the plasma frequency of the background plasma. If turbulence is strong enough to collapse to solitons with scale $D<c / \omega_{p}$, the Compton-boosted beam radiation comes out at even higher frequencies $\sim \gamma_{b}^{2} c / D$ (Weatherall \& Benford 1991). In either case, the radiation frequency is significantly boosted relative to the plasma frequency of the background plasma. We consider this for the Crab pulsar in Sec. 6.2.

\section{A.3. Cycloton instability emission}

In this model, a relativistic particle beam drives a cyclotron instability through the cyclotron resonance, $\omega-k_{\|} v_{\|}-s \omega_{B} / \gamma=0$. Initially the $s=1$ resonance was invoked as a source of Alfvén waves that could scatter particles to higher pitch angles as they passed into the wind, thus enabling synchrotron radiation (Machabeli \& Usov 1979) from the wind nebula.

Later authors, including Kazbegi et al. (1991) and Lyutikov et al. (1999), suggested the anomalous cyclotron instability $(s<0)$ could be a direct radiation source, generating transverse waves which escape the plasma without needing mode conversion. This interaction causes a beam particle to increase its perpendicular momentum, at the same time that it emits a photon; the energy of course comes from the parallel momentum. If wave modes in the background plasma are circularly polarized - as is assumed in their formulation - the emitted radiation is also circularly polarized.

In practice, this model is challenged in application to real pulsars, because of the weak magnetic fields and high plasma densities needed in order to put the emitted radiation in the radio band. It is also challenged by the lack of a clear path to produce short-lived nanoshots. We consider this model for the Crab pulsar in Sec. 6.3. 


\section{A.4. Linear acceleration emission}

In this model, a beam of relativistic charges moving along a magnetic field passes through and responds to an oscillating, parallel electric field. The particles radiate due to the acceleration caused by $E_{\|}$. Melrose (1978) treated this process in the linear limit (assuming $\gamma$ stays nearly constant), and showed that the radiation comes out around $\gamma^{2} \omega_{o}$, where $\omega_{o}$ is the oscillation frequency of the $\mathrm{E}$ field. The radiation is linearly polarized parallel to the projected B field. To reach high brightness temperatures while retaining the singleparticle linear analysis, Melrose suggested negative absorption (maser amplification), but found the optical depth is much less than unity for typical pulsar conditions.

The origin of the oscillating E field is unclear. Melrose (1978) suggested oscillation at $\sim \omega_{p}$, a case which is very similar to free-electron maser models when the wigglers are self-generated Langmuir waves. Unsteady pair creation in an unshielded gap has also been suggested as the source of oscillating E fields (e.g. Levinson et al. 2005, Timohkin \& Arons 2013). These models are still being developed, and the time signature of the unshielded E field is not yet clear. In addition, recent work has explored electric fields strong enough to change the particle's Lorentz factor significantly in one oscillation. This limit seems more relevant to high-enegy emission, and different treatments are still being explored in the literature (Melrose et al. 2009, Reville \& Kirk 2010). We find this mechanism interesting, but further work is needed before it can be usefully tested against pulsar data.

\section{A.5. Maser amplification of radio beams}

Lyubarskii \& Petrova (1996) considered stimulated Compton scattering of pulsar radio emission by the magnetospheric pair plasma in the presence of background radiation. They were interested in scattering out of the radio beam, to explain low-frequency spectral turnovers seen in many pulsars. However in later papers, Petrova (e.g., 2008, 2009) suggested that stimulated scattering of radio emission out of the primary beam can be the origin of secondary components in a pulsar's mean profile (e.g. the interpulse found in a few radio pulsars, or the several components of the Crab's mean profile). Unfortunately, the scattering opacity is hard to predict. It depends not just on magnetospheric parameters, but also varies with scattering regimes, so that the nature of the scattered radiation is extremely sensitive to assumed details of the scattering. While these mod-

els have some nice physics, the difficulty of quantifying the scattering opacity seems to preclude any robust test of them against the data.

Weatherall (2001) also considered stimulated Compton scattering of a pulsar radio beam, but by strong turbulence in the magnetospheric plasma which the beam traverses. He mostly studied scattering in a nonmagnetized plasma, but did include a simple model restricting charge motion to one dimension (appropriate for a high magnetic field). He finds that interesting amplification is possible if the plasma turbulence extremely strong (e.g. turbulent energy density on the order of $10^{12}$ times the plasma temperature). However, he presents no discussion of how such strong turbulence can be maintained, nor does he consider radiation emitted by the turbulence itself (as in Sec. A.1 above).

In addition, both of these models assume the existence of an initial radio beam that can be amplified by stimulated scattering. The origin of the beam is not discussed. In that sense, neither of these models addresses the primary radio emission mechanism. Given the many uncertainties, neither model can easily be compared to radio data. We do not pursue these any further. 


\section{A.6. Coherent charge bunches}

Curvature emission by coherent bunches was one of the first models proposed for pulsar radio emission (e.g. Ruderman \& Sutherland 1975, Buschauer \& Benford 1976). The general idea is that some instability groups $N \gg 1$ charges into a coherently moving bunch that acts as a single large charge, $Q=N e$; this bunch then radiates curvature emission in the radio band. Although this model initially seemed attractive, subsequent analysis (e.g., Melrose 1978, 1995, and references therein) identified several serious problems, including but not limited to the lack of an effective mechanism for forming the bunches, and the inability of the putative charge bunch to hold together long enough to radiate.

This model has recently been revived by Melikidze et al. (2000; also Gil et al. 2004). They follow Karpman et al. (1975), who argued that Langmuir solitons will be chargeseparated if the two charge species have different masses. Melikidze et al. (2000) proposed that solitons in a pair plasma will also be charge-separated if the electrons and positrons have different relativistic streaming speeds. These authors then assumed that the solitons are sufficiently long-lived for the separated "charges" within each soliton to produce curvature emission. They further assumed that the plasma and soliton parameters are just what is needed to put the radiation in the radio band, and they ignored any other radiation caused by plasma dynamics within the soliton. Given the large number of assumptions required by this model, and the lack of any demonstration of the solitons' dynamic evolution, we do not consider this model any further.

\section{A.7. Curvature maser emission}

While simple curvature emission cannot support maser action (Blandford 1975), signal amplification is possible if particle curvature drift and/or field line distortion is taken into account (e.g. Luo \& Melrose 1992, Luo \& Melrose 1994, Lyutikov et al. 1999). However, the model details are very sensitive to the local field line curvature, which is poorly known in the outer magnetosphere. The situation is also complicated by the fact that the energetic particle beam necessary for either type of curvature maser also drives streaming instabilities that can lead to relativistic plasma emission (see Melrose 1995). Furthermore, there is still disagreement in the literature about the efficacy of this mechanism (e.g., Kaganovich \& Lyubarsky 2010). For all of these reasons, we do, not pursue this mechanism here.

\section{Appendix B. Double plasma resonance model for pulsar emission}

The spectral emission bands in the High-Frequency Interpulse have not been seen in any other pulsar. A variety of new models, specific to the emission bands, have been suggested since their discovery, but none have successfully explained all the data. We review several such models in Hankins et al. (2016); here we only discuss one, the Double Plasma Resonance which may explain the "zebra" emission bands seen in Type IV solar flares (e.g., Kuijpers 1975, Zhelezniakov \& Zlotnik 1975, Winglee \& Dulk 1986).

This is another two-stage process. A maser instability generates upper hybrid waves at the cyclotron resonance, and those waves must be converted to transverse modes in order to escape the system. The instability growth is especially rapid at a set of discrete frequencies,

$$
\nu_{\mathrm{res}}=\left(\nu_{\mathrm{p}}^{2}+\nu_{\mathrm{B}}^{2}\right)^{1 / 2}=s \nu_{\mathrm{B}}
$$

where $s$ is the harmonic number, $\nu_{\mathrm{p}}$ is the plasma frequency, $\nu_{\mathrm{B}}$ is the electron cyclotron frequency, and the growth is fastest for waves propagating across the local magnetic field.

If the plasma is non-uniform, with the magnetic field and plasma density varying on 
different scales, the resonance for each harmonic $s$ will be satisfied at a different spatial location, and multiple spectral bands will be seen. This is a stringent constraint. In order to produce a particular value of the band spacing, $\Delta \nu / \nu$, the local structure of the plasma density and magnetic field must have just the right behavior. From equation (B1), in the limit $\nu_{\mathrm{B}} \ll \nu_{\mathrm{p}}$, the spacing between adjacent bands is usually written

$$
\frac{\Delta \nu}{\nu} \simeq \frac{L_{\mathrm{B}}}{s L_{\mathrm{B}}-(s+1) L_{\mathrm{n}}}
$$

where $L_{\mathrm{B}}$ and $L_{\mathrm{n}}$ are the gradient scales of the magnetic field and density: $L_{\mathrm{B}}=B /|\nabla B|$ and $L_{\mathrm{n}}=2 n /|\nabla n|$. For general field and density distributions, $L_{\mathrm{B}}$ and $L_{\mathrm{n}}$ are functions of position, and equation $(\overline{B 2})$ becomes an implicit condition for the location at which equation (B 1) is satisfied for a given harmonic $s$.

In addition to the geometrical restriction, this theory has other important requirements on the plasma. The magnetic field must be weak, $\nu_{\mathrm{B}} \ll \nu_{\mathrm{p}}$, and much lower than is predicted anywhere in the standard magnetosphere if $\nu_{\text {res }}$ is to lie in the radio band. The theory has only been developed for non-relativistic, electron-ion plasmas. Zheleznyakov et al. (2012) suggested the instability may also develop in pair plasmas, but we have not found that work in the literature. There must be an excess of energetic particles moving across the magnetic field - a situation which is common in solar coronal loops, but not expected in the standard magnetosphere.

Despite the challenges, we find this model attractive, because it offers a clear physical cause of discrete spectral bands such as those we see in the High-Frequency Interpulse of the Crab pulsar. We discuss its relevance for the Crab pulsar in Sec. 7.3

\section{REFERENCES}

Abdo, A. A., Ackerman, M., Ajello, M. et al. 2010 Fermi Large Area Telescope observations of the Crab pulsar and Nebula. Astrophys. J. 708, 1254-1267.

Abdo, A. A., Ajello, M., Allafort et al. 2013 The second Fermi Large Area Telescope catalog of gamma-ray pulsars. Astrophys. J. Supp. 208, 17.

Allen, M. C. \& Melrose, D. B. 1982 Elliptically polarized natural modes in pulsar magnetospheres. Proc. Ast. Soc. of Australia, 4, 365-370.

Arendt, P. N. Jr. \& Eilek, J. A. 2002 Pair creation in the pulsar magnetosphere. Astrophys. J. 581, 451-469.

Arons, J. \& Barnard, J. J. 1986 Wave propagation in pulsar magnetospheres - dispersion relations and normal modes of plasmas in superstrong magnetic fields. Astrophys. J. 302 $120-137$.

Arons, J. \& Scharlemann, E. T. 1979 Pair formation above pulsar polar caps - structure of the low altitude acceleration zone. Astrophys. J. 231, 854-879.

Bai, X.-N. \& Spitkovsky, A. 2010 Modeling of gamma-ray pulsar light curves using the force-free magnetic field. Astrophys. J. 715, 1282-1301.

Baker, D. N., Borovsky, J. E., Benford, G. \& Eilek, J. A. 1988 The collective emission of electromagnetic waves from astrophysical jets: luminosity gaps, BL Lacertae objects, and efficient energy transport. Astrophys. J. 326, 110-124.

Benford, G. 1992 Broadband microwave generation by beam-plasma turbulence. IEEE Trans. Plasma Sci. 20, 370-372.

Benford, G. \& Tzach, D. 2000 Coherent synchroton emission observed: implications for radio astronomy. Monthly Notices Royal Ast. Soc. 317, 497-500.

Blandford, R. D. 1975 Amplification of radiation by relativistic particles in a strong magnetic field. Astrophys. J. 170, 551-557.

Bogovalov, S. V. 1999 On the physics of cold MHD winds from oblique rotators. Astron. Astrophys. 349, 1017-1026. 
Bucciantini, N., Arons, J. \& Amato, E. 2010 Modelling spectral evolution of pulsar wind nebulae inside supernova remnants. Monthly Notices Royal Ast. Soc. 410 381-389.

Buschauer, R. \& Benford, G. 1976 General theory of coherent curvature radiation. Monthly Notices Royal Ast. Soc. 177, 109-136.

Carlqvist, P. 1982 On the physics of relativistic double layers. Astrophys. ES Space Sci. 87, 21-39.

Cerutti, B., Philippov, A., Parfrey, K. \& Spitkovsky, A. 2015 Particle acceleration in axisymmetric pulsar current sheets. Monthly Notices Royal Ast. Soc. 448, 606-619.

Chen, B., Bastian. T. S., Gary, D. E. \& Jing, J. 2011 Spatially and spectrally resolved observations of a zebra pattern in a solar decimetric radio burst. Astrophys. J. 736, 64.

Cheng, K. S., Ho, D. \& Ruderman, M. 1986 Energetic radiation from rapidly spinning pulsars. I - outer magnetosphere gaps. Astrophys. J. 300, 500-521.

Cheng, K. S., Ruderman, M. \& Zhang, L. 2000 A three-dimensional outer magnetospheric gap model for gamma-ray pulsars: geometry, pair production, emission morphologies, and phase-resolved spectra. Astrophys. J. 537, 964-976.

Chernov, G. P., Yan, Y. H., Fu, Q. J. \& Tan, Ch. M. 2005 Recent data on zebra patterns. Astron. Astrophys. 437, 1047-1054.

Contopoulos, I. \& Kalapotharakos, C. 2010 The pulsar synchrotron in 3D: curvature radiation. Monthly Notices Royal Ast. Soc. 404, 767-778.

Crossley, J. H., Eilek, J. A., Hankins, T. H. \& Kern, J. S. 2010 Short-lived radio bursts from the Crab pulsar. Astrophys. J. 722, 1908-1920.

Deutsch, A. J. 1955 The electromagnetic field of an idealized star in rigid rotation in vacuo. Annales d'Astrophysique, 18, 1-10.

Dyks, J., Harding, A. K. \& Rudak, B. 2004 Relativistic effects and polarization in three highenergy pulsar models. Astrophys. J. 606, 1125-1142.

Fung, P. K. \& Kuijpers, J. 2002 A free-electron laser in the pulsar magnetosphere. Astron. Astrophys. 422, 817-830.

Gil, J., Lyubarsky, Y. \& Melikidze, G. I. 2004 Curvature radiation in pulsar magnetospheric plasma. Astrophys. J. 600, 872-882.

Goldreich, P. \& Julian, W. H. 1969 Pulsar electrodynamics. Astrophys. J. 157, 869-880.

Hankins, T. H., Kern, J. S., Weatherall, J. C. \& Eilek, J. A. 2003 Microsecond radio bursts from strong plasma turbulence in the Crab pulsar. Nature 422, 141-143.

Hankins, T. H. \& Eilek, J. A. 2007 Radio emission signatures in the Crab pulsar. Astrophys. J. 670, 693-701.

Hankins, T. H., Jones, G. \& Eilek, J. A. 2015 The Crab pulsar at centimeter wavelengths. 1. Ensemble Characteristics. Astrophys. J. 802, 130.

Hankins, T. H., Eilek, J. A. \& Jones, G. 2016 The Crab pulsar at centimeter wavelengths. 2. Single pulse characteristics. Submitted to Astrophys. J.

Hibschman, J. A. \& Arons, J. 2001 Pair production multiplicities in rotation-powered pulsars. Astrophys. J. 560, 871-884.

Ho, C. 1989 Spectra of Crab-like pulsars. Astrophys. J. 342, 396-405.

Kaganovich, A. \& Lyubarsky, Y. 2010 Curvature-drift instability fails to generate pulsar radio emission. Astrophys. J. 721, 1164-1173.

Kalapotharakos, C., Contopoulos, I. \& Kazanas, D., 2012a The extended pulsar magnetosphere. Monthly Notices Royal Ast. Soc. , 420, 2793-2798.

Kalapotharakos, Kazanas, D., Harding, A. \& Contopoulos, I. 2012b Toward a realistic pulsar magnetosphere. Astrophys. J. 749, 2.

Karpman, V. I., Norman, C. A., Ter Harr, D. \& Tsytovich, V. N. 1975 Relativistic solitons and pulsars. Phys. Scripta 11, 271-274.

Kato, K. G., Benford, G. \& Tzach, D. 1983 Detailed spectra of high-power broadband microwave radiation from interactions of relativistic electron beams with weakly magnetized plasmas. Physics of Fluids 26, 3636-3649.

Kazbegi, A. K., Machabeli, G. Z. \& Melikidze, G. I. 1991 On the circular polarization in pulsar emission. Monthly Notices Royal Ast. Soc. 253, 377-387.

Kuijpers J. 1975 A unified explanation of solar type IV DM continua and ZEBRA patterns. Astron. Astrophys. 40, 405-410.

Kuijpers, J. 1990 Coherent radiation from electrostatic double layers, in Plasma Phenomena 
in the Solar Atmosphere, ed. M. Dubois, F. Bely-Dubau \& D. Grèsellon (Les Editions de Physique, France) p. 17.

Kunzl, T., Lesch, H., Jessner, A. \& von Hoensbroech, A. 1998 On pair production in the Crab pulsar. Astrophys. J. 505, L139-L141.

LaBelle, J., Truemann, R. A., Yoon, P. H. \& Karlický, M. 2003 A model of zebra emission in solar Type IV radio bursts. Astrophys. J. 593, 1195-1207.

Ledendev, V. G., Karlický, M., Yan, Y. \& Fu, Q. An estimation of the coronal magnetic field strength from spectrographic observations in the microwave range. Solar Phys. 202, 71-79.

Levinson, A., Melrose, D., Judge, A. \& Luo, Q. 2005 Large-amplitude, pair-creating oscillations in pulsar and black hole magnetospheres. Astrophys. J. 631, 456-465.

Li, J., Spitkovsky, A. \& Tchekhovskoy, A. 2012 Resistive solutions for pulsar magnetospheres. Astrophys. J. 746, 60.

Luo, Q. \& Melrose, D. B. 1992 Coherent curvature emission and radio pulsars. Monthly Notices Royal Ast. Soc. 258, 616-620.

Luo, Q. \& Melrose, D. B. 1995 Curvature maser emission due to field line torsion in pulsar magnetospheres. Monthly Notices Royal Ast. Soc. 276, 372-382.

Lyubarskii, Yu. E. \& Petrova, S. A. 1996 Stimulated scattering of radio emission in pulsar magnetospheres. Astr. Lett., 22, 399-408.

Lyutikov, M., Machabeli, G. \& Blandford, R. 1999 Cherenkov-curvature radiation and pulsar radio emission generation. Astrophys. J. 512, 804-826.

Machabeli, G. Z. \& Usov, V. V. 1979 Cyclotron instability in the magnetosphere of the Crab Nebula pulsar, and the origin of its radiation. Soviet Ast. Lett. 5, 238-241.

Melikidze, G. I., Gil, J. A. \& Pataraya, A. D. 2000 The spark-associated soliton model for pulsar radio emission. Astrophys. J. 544, 1081-1096.

Melrose, D. B. 1978 Amplified linear acceleration emission applied to pulsars. Astrophys. J. 225, $557-573$.

Melrose, D. B. 1995 The models for radio emission from pulsars - the outstanding issues. J. Astrophys. Astr. 16, 137-164.

Melrose, D. B., Rafat, M. Z. \& Luo, Q. 2009 Linear acceleration emission I: motion in a largeamplitude electrostatic wave. Astrophys. J. 698, 115-123.

Melrose, D. B. \& Gedalin, M. E. 1999 Relativistic plasma emission and pulsar radio emission: a critique. Astrophys. J. 521, 351-361.

Moffett, D. A. \& Hankins, T. H. 1996 Multifrequency radio observations of the Crab pulsar. Astrophys. J. 468, 779-783.

Moffett, D. A. \& Hankins, T. H. 1999 Polarimetric properties of the Crab pulsar between 1.4 and $8.4 \mathrm{GHz}$. Astrophys. J. 522, 1046-1052.

Muslimov, A. G. \& Harding, A. K. High-altitude particle acceleration and radiation in slot gaps. Astrophys. J. 606, 1143-1153.

Ng, C.-Y. \& Romani, R. W. 2004 Fitting pulsar wind tori. Astrophys. J. 601, 479-484.

Qiao, G. J., Lee, K. J., Wang, H. G. et al. 2004 The inner annular gap for pulsar radiation: gamma-ray and radio emission. Astrophys. J. 606 L49-L52.

Pétri, J. \& Kirk, J. G., 2005 The polarization of high-energy pulsar radiation in the striped wind model. Astrophys. J. 627, L37-L40.

Petrova, S. A. 2008 Interpretation of the low-frequency peculiarities in the radio profile structure of the Crab pulsar. Monthly Notices Royal Ast. Soc. 385, 2143-2150.

Petrova, S. A. 2009 Formation of the radio profile components of the Crab pulsar. Monthly Notices Royal Ast. Soc. 395, 1723-1732.

Radhakrishnan, V. \& Cooke, D. J. 1969 Magnetic poles and the polarization structure of pulsar radiation. Astrophys Lett., 3, 225-229.

Rankin, J. M., Comella, J. M., Craft, H. D. Jr. et al. 1970 Radio pulse shapes, flux densities, and dispersion of pulsar NP 0532. Astrophys. J. 162, 707-725.

Rickett, B. J. 1975 Amplitude-modulated noise: an empirical model for the radio radiation received from pulsars. Astrophys. J. 197, 185-191.

Reville, B. \& Kirk, J. G. 2010 Linear acceleration emission in pulsar magnetospheres. Astrophys. J. 715, 186-193.

Robinson, P. A. 1977 Nonlinear wave collapse and strong turbulence. Rev. Mod. Phys. 69, 507572 . 
Romani, R. W. \& Yadigaroglu, I.-A. 1995 Gamma-ray pulsars: emission zones and viewing geometries. Astrophys. J. 438 314-321.

Ruderman, M. A. \& Sutherland, P. G. 1975 Theory of Pulsars - Polar caps, sparks, and coherent microwave radiation. Astrophys. J. 196, 51-72.

Schopper, R., Ruhl, H., Kunzl, T. A. \& Lesch, H. 2003 Kinetic simulation of the coherent radio emission from pulsars. Laser and Particle Beams 21, 109-113.

Shearer, A., Collins, S., Naletto, G. et al. 2012 High-time-resolution optical observations of the Crab pulsar; in Electromagnetic radiation from pulsars and magnetars ed. W. Lewandoski, O. Maron, J. Kijak \& A. Słowikowska (San Francisco: Astronomical Society of the Pacific), $11-14$.

Shcherbakov, R. V. 2008 Propagation effects in magnetized transrelativistic plasmas. Astrophys. J. 688, 695-700.

Słowikowska, A., Kanbach, G., Kramer, M. \& Stefanescu, A. 2009 Optical polarization of the Crab pulsar: precision measurements and comparison to the radio emission. Monthly Notices Royal Ast. Soc. 397, 103-123.

Timokhin, A. N. \& Arons, J. 2013 Current flow and pair creation at low altitude in rotationpowered pulsars' force-free magnetospheres: space charge limited flow. Monthly Notices Royal Ast. Soc. 429, 20-54.

Weatherall, J. C. 1997 Modulational instability, mode conversion and radio emission in the magnetized pair plasma of pulsars. Astrophys. J. 483, 402-413.

Weatherall, J. C. 1998 Pulsar radio emission by conversion of plasma wave turbulence: nanosecond time structure. Astrophys. J. 506, 341-346.

Weatherall, J. C. 2001 A relativistic-plasma Compton maser. Astrophys. J. 559, 196-200.

Weatherall, J. C. \& Benford, G. 1991 Coherent radiation from energetic electron streams via collisionless bremsstrahlung in strong plasma turbulence. Astrophys. J. 378, 543-549.

Windsor, R. A. \& Kellog, P. J. 1974 Polarization of inverse plasmon scattering. Astrophys. J. 190, 167-173.

Winglee, R. M. \& Dulk, G. A. 1986 The electron-cyclotron maser instability as a source of plasma radiation. Astrophys. J. 307, 808-819.

Zampieri, L., Cadez, A., Barbiere, C. et al. 2014 Optical phase coherent timing of the Crab nebula pulsar with Iqueye at the ESO New Technology Telescope. Monthly Notices Royal Ast. Soc. 439, 2813-2821.

Zhelezniakov, V. V. \& Zlotnik, E. Ia. 1975 Cyclotron wave instability in the corona and origin of solar radio emission with fine structure. I - Bernstein modes and plasma waves in a hybrid band. Solar Phys. 43, 431-451.

Zheleznyakov, V. V., Zaitsev, V. V. \& Zlotnik, E. Ya. 2012 On the analogy between the zebra patterns in radio emission from the sun and the Crab pulsar. Ast. Lett. 9 589-604. 